ARTICLE

https://doi.org/10.1038/s41467-019-12855-6

\title{
Clathrin light chain A drives selective myosin VI recruitment to clathrin-coated pits under membrane tension
}

Matteo Biancospino 1,6, Gwen R. Buel (10 2,6, Carlos A. Niño 1,6, Elena Maspero, Rossella Scotto di Perrotolo1, Andrea Raimondi (10 ${ }^{3}$, Lisa Redlingshöfer ${ }^{4}$, Janine Weber ${ }^{1}$, Frances M. Brodsky ${ }^{4 \star}$, Kylie J. Walters ${ }^{2 \star} \&$ Simona Polo (1) ${ }^{1,5 *}$

Clathrin light chains (CLCa and CLCb) are major constituents of clathrin-coated vesicles. Unique functions for these evolutionary conserved paralogs remain elusive, and their role in clathrin-mediated endocytosis in mammalian cells is debated. Here, we find and structurally characterize a direct and selective interaction between CLCa and the long isoform of the actin motor protein myosin $\mathrm{VI}$, which is expressed exclusively in highly polarized tissues. Using genetically-reconstituted Caco-2 cysts as proxy for polarized epithelia, we provide evidence for coordinated action of myosin VI and CLCa at the apical surface where these proteins are essential for fission of clathrin-coated pits. We further find that myosin VI and Huntingtininteracting protein 1-related protein (Hip1R) are mutually exclusive interactors with CLCa, and suggest a model for the sequential function of myosin VI and Hip1R in actin-mediated clathrin-coated vesicle budding.

\footnotetext{
${ }^{1}$ IFOM, Fondazione Istituto FIRC di Oncologia Molecolare, 20139 Milan, Italy. ${ }^{2}$ Structural Biophysics Laboratory, Center for Cancer Research, National Cancer Institute, Frederick, MD 21702, USA. ${ }^{3}$ Experimental Imaging Center, San Raffaele Scientific Institute, Milan, Italy. ${ }^{4}$ Division of Biosciences, University College London, London WC1E 6BT, UK. ${ }^{5}$ Dipartimento di Oncologia ed Emato-oncologia, Universita' degli Studi di Milano, 20122 Milan, Italy. ${ }^{6}$ These authors contributed equally: Matteo Biancospino, Gwen R. Buel, Carlos A. Niño. *email: f.brodsky@ucl.ac.uk; kylie.walters@nih.gov; simona.polo@ifom.eu
} 
C lathrin-coated vesicles (CCVs) are well-characterized molecular machines, responsible for the internalization of nutrients, signaling receptors, and adhesion molecules from the plasma membrane (PM) as well as for cargo transport between the trans-Golgi network and endosomes ${ }^{1,2}$. Clathrin is a three-legged molecule (triskelion), composed of three clathrin heavy chain (CHC) and three clathrin light chain (CLC) subunits. The clathrin triskelia assemble around a budding vesicle, creating a characteristic polyhedral coat, forming a clathrin-coated pit (CCP) that captures protein cargo and further matures into a CCV.

While CHCs are essential components of the cages, CLCs are apparently more regulatory. Functional roles for CLCs have been linked to regulation of cage rigidity, assembly and disassembly by extrapolation from in vitro studies ${ }^{1}$, and to clathrin-mediated endocytosis (CME) of model cargos, including G-protein-coupled receptors $^{3-5}$. Importantly, CLCs appear essential at the apical surface of highly polarized tissues, where membrane tension is higher than normal and actin dynamics is critical for membrane invagination and fission ${ }^{6}$. This process involves the conserved interaction between CLCs and the actin-remodeling huntingtininteracting proteins (Hipl and Hip1R) ${ }^{7,8}$, an interaction that also contributes to clathrin-actin interactions during endosomal recycling and is needed for cell migration ${ }^{9}$. CLCs are also required for uptake of large particles, including viruses and pathogenic bacteria, such as Listeria, Yersinia, and Salmonella ${ }^{10-12}$. In these cases, the direct association of CLC with actin remodelers distinguishes these clathrin-dependent processes from canonical CME. Finally, CLCs, together with Hip1R and the actin motor protein myosin $\mathrm{VI}^{13}$, participate in cell-cell junction formation and remodeling through molecular mechanisms that have not been defined ${ }^{14}$.

In vertebrates, two genes encode the CLCa and CLCb variants, which are $40 \%$ divergent in sequence. High conservation of each protein variant in all vertebrate species suggests that they have distinct functions beyond their shared interaction with the Hip proteins. However, evidence for CLCa- and CLCb-specific roles is limited. CLCa was shown to be preferentially involved in cell spreading and migration ${ }^{15}$, whereas maturation of CCPs from flat lattices to invaginated buds requires phosphorylation of CLCb ${ }^{16}$. $\mathrm{CLCb}$ was also found to be overexpressed in non-small-cell lung cancer, causing altered CCP dynamics and consequently aberrant growth factor signaling ${ }^{17}$.

In a previous study, we identified clathrin as a major and specific binding partner of myosin VI isoforms that contain the a2-linker (named myosin $\mathrm{VI}_{\text {long }}$ ) and we found that myosin $\mathrm{VI}_{\text {long }}$ localizes to CCPs independently of the presence of the endocytic adaptor Dab-2 ${ }^{18}$. These findings suggested a molecular link between clathrin and myosin $\mathrm{VI}_{\text {long. }}$. In this manuscript, we now demonstrate that this interaction is due to a direct and selective interaction of myosin $\mathrm{VI}_{\text {long }}$ with CLCa and not CLCb. We use nuclear magnetic resonance (NMR) spectroscopy to solve the structure of the myosin $\mathrm{VI}_{\text {long: }}$ :CLCa complex and characterize the mechanism of interaction. The CLCa-binding region in myosin VI partially overlaps with the ubiquitin-binding domain MyUb $^{19}$ while CLCa binds myosin VI with a surface that is in close proximity to the Hip1R-binding site ${ }^{7}$. Guided by the structure, we identify a CLCa point mutant that loses myosin VIbinding ability, while leaving unaltered its capacity to interact with $\mathrm{CHC}$ or Hip1R. We further investigate the biological implications of these molecular interactions, taking advantage of genetically reconstituted Caco- 2 cells growing in three dimension (3D) as cysts. Altogether, our results indicate that the interaction between myosin $\mathrm{VI}_{\text {long }}$ and CLCa is needed to generate the force that leads to invagination and fission of CCPs at the apical surface of highly polarized cells and suggests a model for the sequential function of myosin VI and Hip1R in actin-mediated coated vesicle budding.

\section{Results}

CLCa is a direct and specific interactor of myosin $\mathrm{VI}_{\text {long. In }}$ myosin VI, the presence of helix a 2 structurally defines a clathrinbinding domain that is unique to myosin $\mathrm{VI}_{\text {long }}{ }^{18,19}$ (Fig. 1a). To identify the corresponding myosin $\mathrm{VI}_{\text {long }}$ interacting region within clathrin, we performed experiments with green fluorescent protein (GFP)- and glutathione S-transferase (GST)- tagged CHC truncations. The results revealed that the $\mathrm{C}$-terminal part of the $\mathrm{CHC}$ is required for myosin VI interaction but failed to demonstrate direct binding between the two proteins (Supplementary Fig. 1a). Given that CLCs are reported to interact with the C-terminus of $\mathrm{CHC}$, we hypothesized their involvement in recruiting myosin VI to CCPs. Corroborating our hypothesis, confocal microscopic analysis of HeLa cells revealed reduced colocalization of myosin $\mathrm{VI}_{\text {long }}$ and $\mathrm{CHC}$ upon CLCa transient knockdown (KD) as measured by Mander's coefficient (Supplementary Fig. 1b, c). Unexpectedly, CLCb KD had the opposite effect, increasing the co-localization levels of $\mathrm{CHC}$ and myosin $\mathrm{VI}_{\text {long }}$, thus suggesting different roles for the two CLCs.

We next examined direct binding in a pull-down assay by using bacterially purified GST-CLCa and GST-CLCb full-length proteins and purified fragments of long and short myosin VI isoforms spanning amino acids 998-1131 (Fig. 1a). The results demonstrated direct and selective binding of CLCa to myosin $\mathrm{VI}_{\text {long }}$ while both CLCs showed minimal binding to myosin $\mathrm{VI}_{\text {short }}$ (Fig. 1b).

To formally test whether CLCa acts as a bridge between myosin $\mathrm{VI}_{\text {long }}$ and $\mathrm{CHC}$, we exploited CLCa knockout (KO) mice that were recently generated ${ }^{5}$ and newly produced $\mathrm{CLCb} \mathrm{KO}$ mice. Wholebrain lysates obtained from CLCa and CLCb KO mice and wildtype (WT) littermates were used in a pull-down assay with GSTmyosin VI ${ }^{998-1131}$ from long and short myosin VI isoforms. Using WT littermates, GST-myosin $\mathrm{VI}_{\text {long }}$ efficiently pulled down $\mathrm{CHC}$ (Fig. 1c). This interaction was dependent on CLCa since clathrin was not detected using brain lysate from CLCa KO animals (Fig. 1c). Of note, brain lysate from CLCb KO mice behaved as WT littermates, similarly showing interaction between clathrin and myosin $\mathrm{VI}_{\text {long }}$ (Fig. 1c), despite lacking CLCb (Fig. 1d).

The lysis conditions used in these experiments and in our previous study ${ }^{18}$ favor free triskelia in solution as opposed to assembled clathrin. During clathrin cage assembly, CLCs undergo conformational changes ${ }^{8}$, and to investigate whether the interaction of the coat protein with myosin VI can occur in the context of a formed CCV, we exploited the ability of clathrin to selfassemble in a $\mathrm{pH}$-dependent manner ${ }^{20,21}$. We purified clathrin from pig brain CCVs and induced the formation of assembled cages from native clathrin containing an endogenous mixture of CLCa and CLCb, CHC with no CLCs, or CHC reconstituted with either CLCa or CLCb only (see Methods and Supplementary Fig. 2a-c for details). Purified myosin $\mathrm{VI}^{998-1131}$ protein cosedimented with native clathrin cages and cages formed from clathrin with only CLCa, whereas only weak sedimentation was observed with CLCb-only cages that was similar to CHC-only cages (Fig. 1e). In summary, these results demonstrate that CLCa is the subunit of clathrin responsible for binding to myosin $\mathrm{VI}_{\text {long, }}$, both in the context of free triskelia and in CCVs.

CLCa and myosin $\mathrm{VI}_{\text {long }}$ interact with sub-micromolar affinity. To obtain biochemical and structural insights into the myosin $\mathrm{VI}_{\text {long:CLCa complex, we systematically designed CLCa deletions }}$ taking into consideration the known interaction domains of the protein $^{22}$ (Fig. 2a). Incubation of these constructs with bacterially purified myosin $\mathrm{VI}^{998-1131}$ identified a region of interaction in CLCa that spans amino acids 47-97 (Fig. 2b). This fragment of clathrin falls in a region of limited conservation between the two 
a

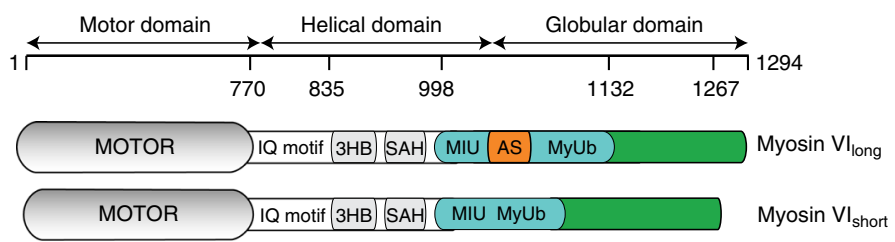

C

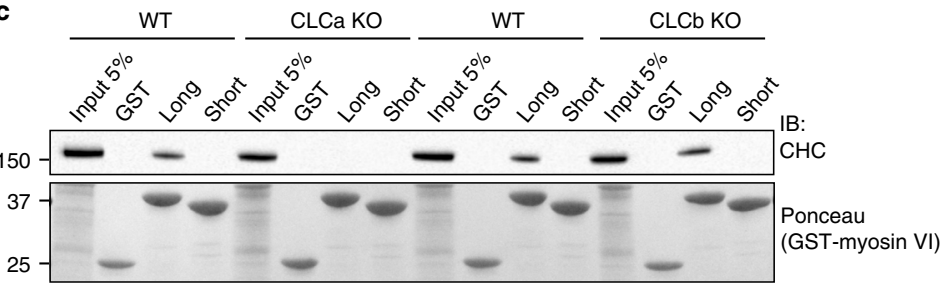

d

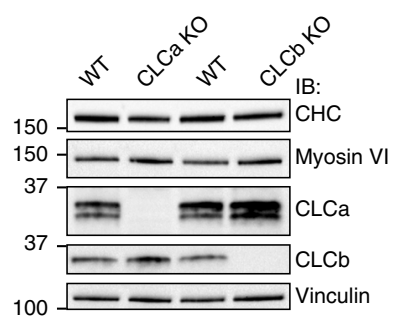

b

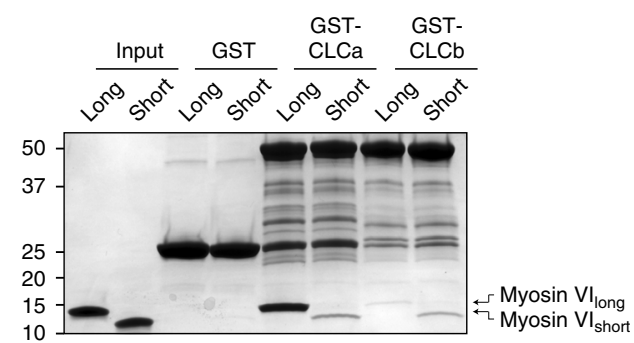

e

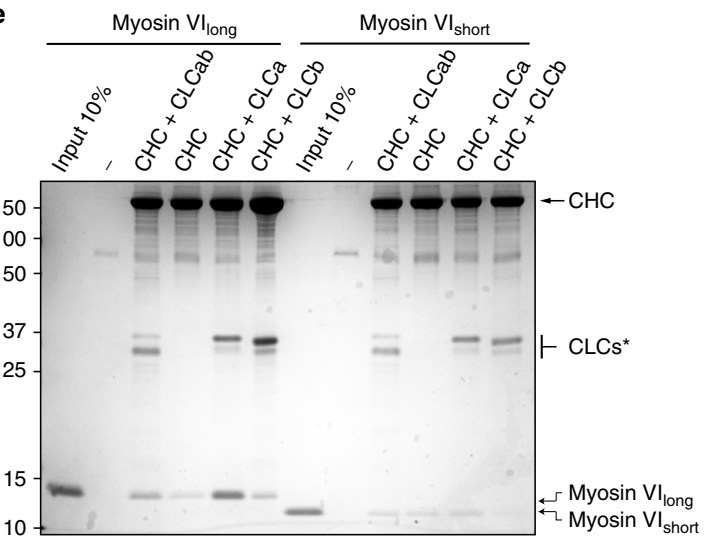

Fig. $1 \mathrm{CLCa}$ is a direct and specific interactor of myosin $\mathrm{VI}_{\text {long }}$ in triskelia and clathrin cages. a Scheme of the myosin $\mathrm{VI}$ highlighting the region involved in clathrin binding (amino acids 998-1131 of the long isoform). Long and short isoforms are reported together with the domains and motifs previously identified, including IQ motif, 3HB (three-helix bundle), SAH (single $\alpha$-helix), MIU (motif interacting with ubiquitin), AS (alternative splicing region), and MyUb (myosin VI ubiquitin-binding domain). In orange is represented the alternatively spliced region codifying for the $\alpha 2$-linker ${ }^{18}$. b Pull-down assay with GST-CLCa and CLCb full-length and cleaved and purified fragments spanning amino acids 998-1131 of long and short myosin VI isoforms. Glutathione sepharose beads coupled to GST and GST-tagged proteins were incubated with myosin VI998-1131. After washes, bound proteins were eluted in Laemmlibuffer, resolved through SDS-PAGE, and stained with Coomassie. c Pull-down assay using the long and short GST-myosin VI998-1131 constructs and brain lysates $(500 \mu \mathrm{g})$ obtained from the indicated mouse strains. After washes, bound proteins were eluted in Laemmli-buffer, resolved through SDS-PAGE, and transferred to a nitrocellulose membrane. Immunoblot (IB) was performed with anti-clathrin heavy-chain antibody. Ponceau detect equal loading of GST proteins. d IB of the brain lysates used in (c), as indicated. e Co-sedimentation assay. Equimolar (1.5 $\mu \mathrm{M})$ amount of myosin VI $998-1131$ and clathrin cages were incubated at $4{ }^{\circ} \mathrm{C}$ for $45 \mathrm{~min}$ in the presence of detergent $(0.1 \%$ Triton X-100) and then pelleted by ultracentrifugation. Precipitated proteins were dissolved in Laemmli-buffer, resolved through SDS-PAGE, and stained with Coomassie. CLCs ${ }^{\star}$ indicates the various CLC proteins. Note that in the native cages CLCs (CHC-CLCab) run at different molecular weight $(\mathrm{mw})$ as they are from pig brain while the human $\mathrm{CLCs}$ used for reconstitution are bacterially produced and cleaved from GST

paralogs (Fig. 2a), thus explaining the binding selectivity of CLCa to myosin $\mathrm{VI}_{\text {long. }}$. By analytical size-exclusion chromatography, the CLCa:47-97myosin VI ${ }^{998-1131}$ sample co-eluted at the expected molecular weight for a 1:1 complex, indicating a stable and strong interaction in solution (Supplementary Fig. 2d). Further truncation analyses narrowed down the minimal interaction surface to a short peptide, with no secondary structure predicted, extending from amino acids. 51 to 61 (Fig. 2a and Supplementary Fig. 2e).

To evaluate the biochemical properties of the interacting CLCa peptide, we measured binding affinity of the myosin VI-CLCa interaction by fluorescent polarization (FP) analysis. We designed three different fluorescein-labeled CLCa peptides (Fig. 2a) that span the minimal binding surface (amino acids 51-61) exclusively or with five amino acids added at the $\mathrm{N}$ (amino acids 46-61) or C (amino acids 51-66) terminal end. FP measurements revealed a tenfold increase in affinity for the peptide 46-61 compared to the other two peptides (Fig. 2c), resulting in a dissociation constant $\left(K_{\mathrm{d}}\right)$ of $0.93 \mu \mathrm{M}$. Thus, in addition to the minimal binding region, amino acids spanning 46-51 conserved between CLCa and CLCb contribute to binding, which may explain the residual interaction observed by both CLCs to myosin $\mathrm{VI}_{\text {short }}$ (Fig. 1b).

We further tested the 46-61 peptide for binding to various myosin VI constructs to better define the interaction boundaries on myosin VI. Confirming our previous results ${ }^{18}$, the long and the short isoforms of myosin VI showed 2 log-fold difference in affinity for CLCa $(0.93 \mu \mathrm{M}$ versus $96 \mu \mathrm{M}$, respectively, Fig. 2c, d). As expected, the myosin VI ${ }^{1069-1131}$ construct that lacks the $\alpha 2$ linker displayed a remarkably reduced $K_{\mathrm{d}}$. Finally, the region encompassing amino acids 1050-1131 of myosin $\mathrm{VI}_{\text {long }}$ showed the highest affinity $(0.4 \mu \mathrm{M}$, Fig. $2 \mathrm{~d})$ and was therefore chosen for the subsequent structural experiments.

CLCa ${ }^{46-61}$ forms a helix in a complex with myosin VI ${ }^{1050-1131}$. To further characterize the myosin VI:CLCa binding interface, we first compared two-dimensional (2D) ${ }^{1} \mathrm{H},{ }^{15} \mathrm{~N}-\mathrm{HSQC}$ spectra of myosin $\mathrm{VI}^{1050-1131}$ before and after addition of the unlabeled CLCa peptide spanning 46-61 (Fig. 3a). Large effects were observed for many amino acids, which were quantified by chemical shift perturbation (CSP) analysis (Supplementary Fig. 3). Residues with CSP values of one standard deviation (SD) above average cluster to three regions in the myosin VI sequence (Supplementary Fig. 3) located at the N-terminal end of a2, N-terminal to $\alpha 3$, and toward the C-terminal end of $\alpha 4$. The CSPs above the mean were mapped onto the previously solved structure of free myosin VI (Fig. 3b), which revealed a localized region focused at the C-terminal half of $\alpha 4$ and the N-terminal region of $\alpha 2$. 
a

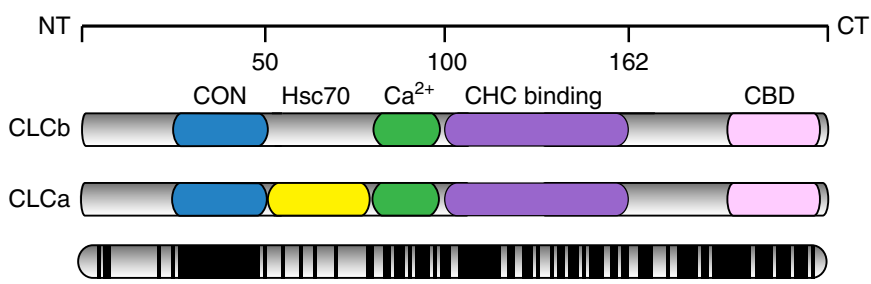

$1-47$

$1-97$

47-97- - - - - - - -

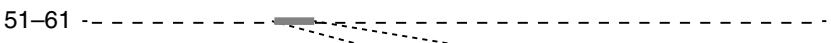

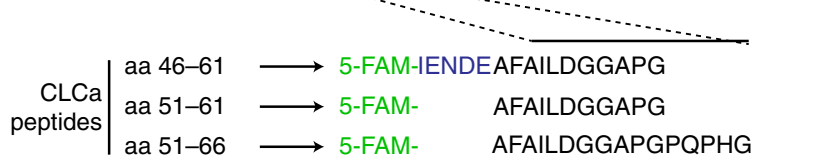

b

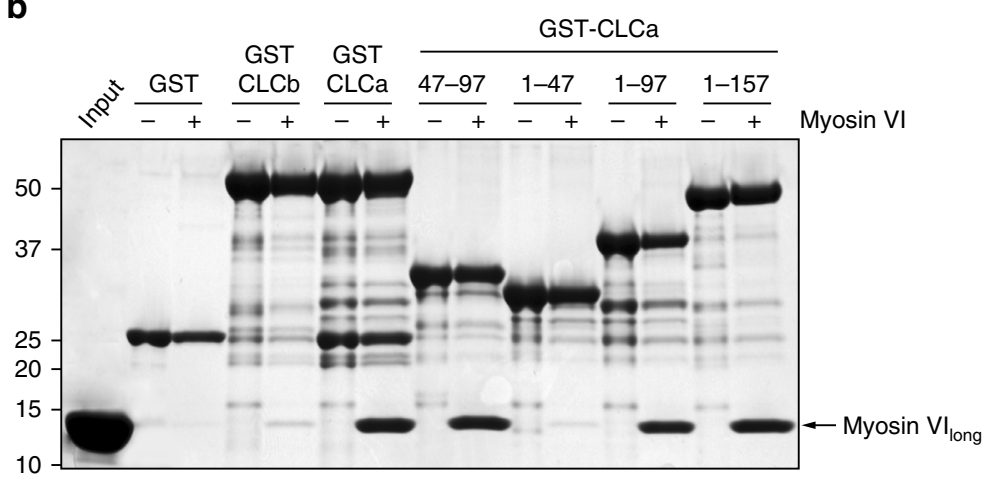

C

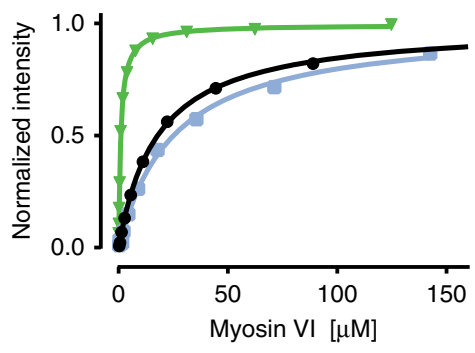

\begin{tabular}{|lc|c|}
\hline $\begin{array}{c}\text { CLCa peptides vs. } \\
\text { myosin } \mathrm{VI}^{998-1131} \text { long: }\end{array}$ & $\mathrm{K}_{\mathrm{d}}$ \\
\hline $46-61$ & - & $0.93 \pm 0.14 \mu \mathrm{M}$ \\
\hline $51-61$ & - & $22.6 \pm 3.97 \mu \mathrm{M}$ \\
\hline $51-66$ & - & $13.1 \pm 4.4 \mu \mathrm{M}$ \\
\hline
\end{tabular}

d

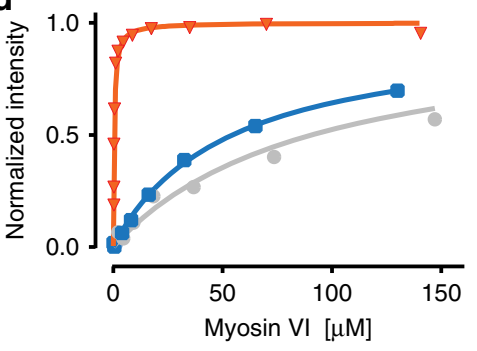

\begin{tabular}{|c|c|}
\hline $\begin{array}{c}\text { CLCa 46-61 peptide vs. } \\
\text { myosin VI constructs: }\end{array}$ & $\mathrm{K}_{\mathrm{d}}$ \\
\hline $998-1131_{\text {short }}--$ & $96 \pm 56 \mu \mathrm{M}$ \\
\hline $1050-1131 \quad-$ & $0.4 \pm 0.7 \mu \mathrm{M}$ \\
\hline $1069-1131 \quad--$ & $22 \pm 3 \mu \mathrm{M}$ \\
\hline
\end{tabular}

Fig. 2 CLCa:myosin VI long interact with sub-micromolar affinity. a Domain structures of CLCa and CLCb. CON conserved Hip-binding region, Hsc70 unique region in $\mathrm{CLC}$ a that stimulates $\mathrm{Hsc70}$ activity in vitro, $\mathrm{Ca}{ }^{2}+\mathrm{EF}-$ hand domain that binds calcium, $\mathrm{CHC}$ binding clathrin heavy chain binding region, $\mathrm{CBD}$ calmodulin-binding domain. Sequence conservation between the two proteins is reported below. Each line represents one amino acid, black line indicates identity. Lower panel, scheme of the selected constructs used in (b) together with the sequence of the three overlapping 5-carboxyfluorescein (5-FAM)conjugated CLCa peptides used for FP analysis in (c). b Pull-down assay with GST-CLCa and CLCb full length and the indicated fragments of CLCa immobilized on glutathione sepharose beads and incubated with the purified fragment spanning amino acids $998-1131$ of myosin $\mathrm{VI}_{\text {long. }}$ After washes, bound proteins were eluted in Laemmli-buffer, resolved through SDS-PAGE, and stained with Coomassie. c FP assay using the three peptides shown in

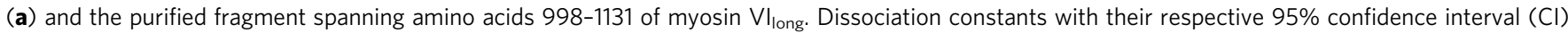
are reported in the table at the bottom. Graph is representative of three independent experiments used to calculate $K_{d}$ and $\mathrm{Cl}$. $\mathbf{d}$ FP assay using peptide 46-61 of CLCa and the indicated fragments of long and short myosin $\mathrm{VI}$ isoforms. Graph, $K_{\mathrm{d}}$, and $\mathrm{Cl}$ as for (c)

In previously published structures of clathrin lattice and pits, the myosin VI-binding region of CLCa identified here was not detectable, as it falls into a flexible region ${ }^{8,23}$. As part of an effort to solve the structure of this CLCa region complexed with myosin $\mathrm{VI}^{1050-1131}$ by NMR, we assigned chemical shift values to the myosin VI-bound CLCa fragment by 3D HNCACB (Supplementary Fig. 4) and nuclear Overhauser effect spectroscopy (NOESY; examples provided in Supplementary Fig. 5) experiments recorded on equimolar ${ }^{15} \mathrm{~N},{ }^{13} \mathrm{C}$-labeled $\mathrm{CLCa}$ and unlabeled myosin VI 1050-1131; we also assigned chemical shift values to CLCa-bound myosin VI, as described in Methods. The Ca and C' chemical shift assignments of CLCa were compared to those of randomly coiled values to generate a chemical shift index plot (Supplementary Fig. 6). This information was combined with NOE analyses obtained by NOESY experiments to define the secondary structure of CLCa (Supplementary Fig. 6) as being a-helical from residues I46-I54.

We next measured intermolecular interactions between myosin VI and CLCa directly by performing $3 \mathrm{D}{ }^{1} \mathrm{H},{ }^{13} \mathrm{C}$ half-filtered
NOESY experiments; resulting spectra show NMR signals between intermolecular atoms that are within $5 \AA$ of each other $^{24}$. In agreement with the CSP analysis, we identified large numbers of intermolecular NOEs between CLCa and helices a2 and $\alpha 4$ of myosin VI (Supplementary Figs. 7 and 8). In particular, residues P1055, M1058, and M1062 from helix a2 (Supplementary Figs. 7a, b and 8) and V1120, Y1121, and W1124 from helix a4 (Supplementary Figs. 7c-f and 8) displayed numerous intermolecular NOEs to CLCa.

CLCa binds to isoform-specific $\boldsymbol{\alpha} 2$ and $\alpha 4$ of MyUb. NMR data (summarized in Table 1), including 221 intermolecular NOE interactions between myosin VI and CLCa, were used to calculate the myosin VI:CLCa structure. The 20 lowest energy structures (Fig. 3c) converged with a backbone root mean square deviation (r.m.s.d.) in the ordered regions of $0.36 \AA$ (Table 1). The myosin VI structure is largely unchanged in the complex, retaining its 
a

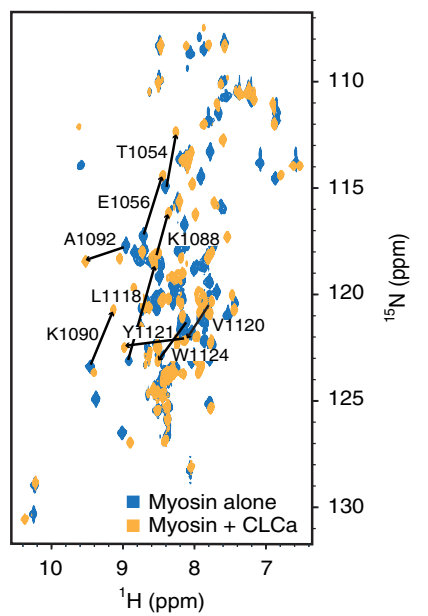

C

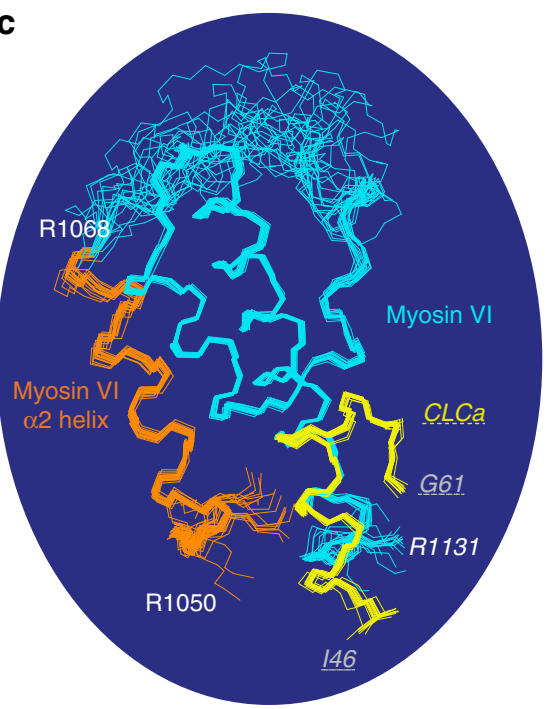

e

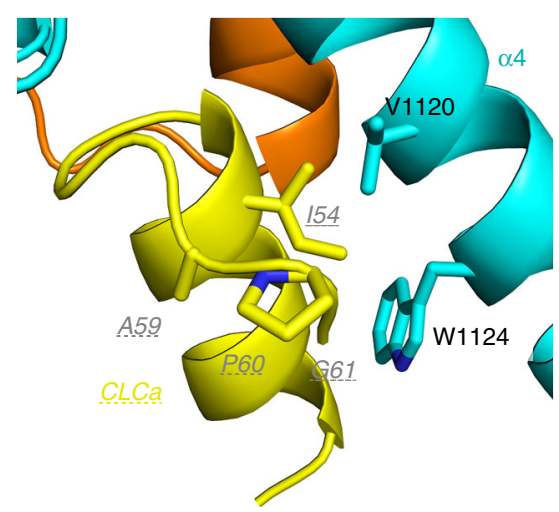

b

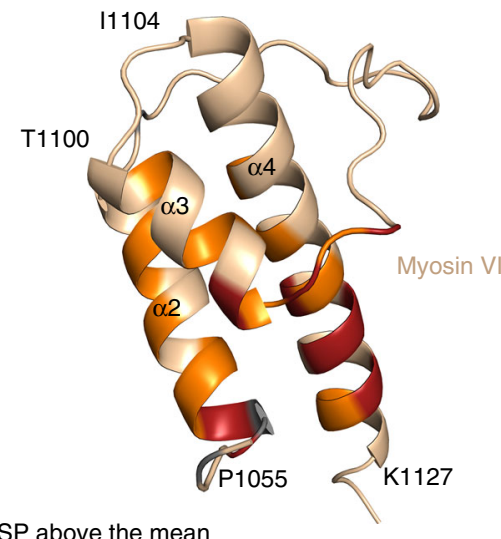

CSP above the mean

CSP $>1$ SD above the mean

d

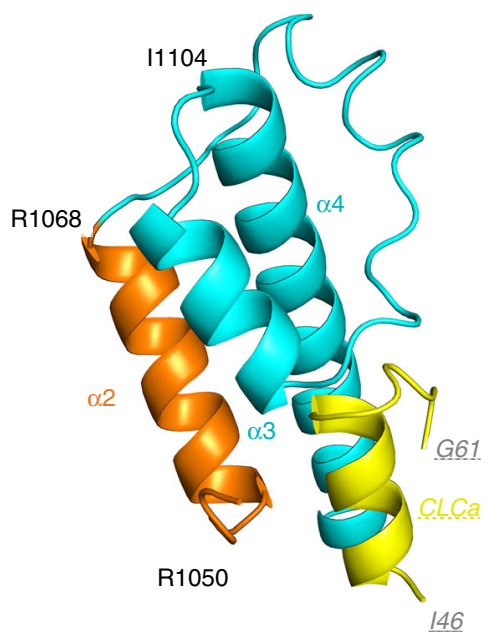

$\mathbf{f}$

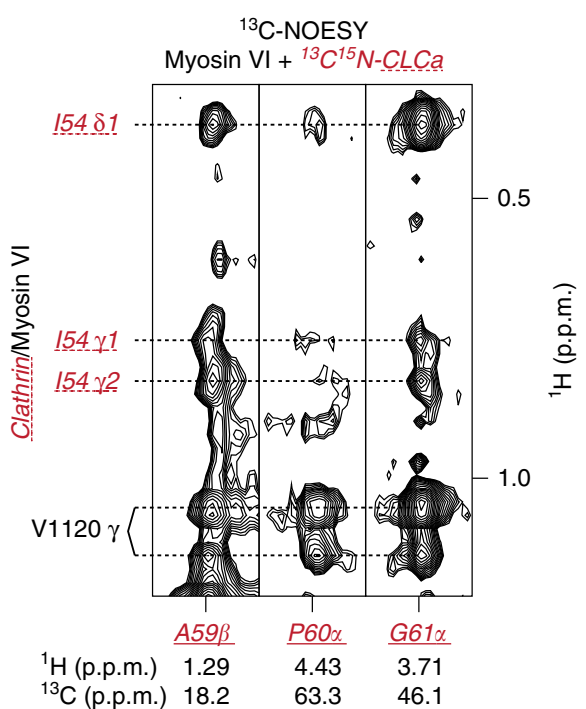

L55-G58 forms a helical turn with A59-G61 folding back along one side of the CLCa $a$-helix (Fig. 3e), a structure supported by NOEs to CLCa I54 and to myosin VI a4 (V1120 and W1124, Fig. $3 f$ and Supplementary Fig. 7c, e). The hydrophobicity of CLCa A59 and P60 likely drives this segment to remain in close contact with the helices (Fig. 3e), as supported by the NMR data (Fig. 3f). three helices and a disordered loop that follows the C-terminal end of isoform-specific a2. In agreement with our chemical shift index data (in Supplementary Fig. 6), $\mathrm{CLCa}^{46-61}$ forms a short $\alpha$ helix (yellow, Fig. 3c-e) that appears to complete the myosin VI structural domain by abutting the $\mathrm{N}$-terminal end of $\alpha 3$ and packing against $\alpha 2$ and $\alpha 4$ (Fig. 3d). At the C-terminal end, CLCa 
Fig. 3 The myosin VI-binding region of CLCa forms an $\alpha$-helix that interlocks with the helices of myosin VI. a $2 \mathrm{D}^{1} \mathrm{H},{ }^{15} \mathrm{~N}-\mathrm{HSQC}$ spectrum of $0.78 \mathrm{mM}{ }^{15} \mathrm{~N}$ labeled myosin VI ${ }^{1050-1131}$ alone (blue) and in the presence of equimolar unlabeled CLCa ${ }^{46-61}$ (orange). Notable changes in NMR signals following addition of CLCa are indicated with arrows and labeled with the assigned amino acid residue from myosin VI. $\mathbf{b}$ On the previously solved structure of myosin VI1050-1131 (PDB 2N12), amino acids with CSPs greater than the mean or 1SD above the mean are indicated in orange or red, respectively. CSPs were calculated according to the definition $\mathrm{CSP}=\left[(0.2 \Delta \delta \mathrm{N})^{2}+(\Delta \delta \mathrm{H})^{2}\right]^{1 / 2}$ where $\Delta \delta \mathrm{N}$ and $\Delta \delta \mathrm{H}$ represent chemical shift differences for the amide nitrogen and proton of each residue, respectively. Residues without amide protons (P1051, P1055, and P1070) are indicated in gray, and those at the beginning and end of each helix are labeled. c Superimposition of the 20 lowest energy structures calculated for myosin VI1050-1131 in complex with CLCa46-61. CLCa is shown in yellow; myosin $\mathrm{VI}$ is colored cyan except for the isoform-specific $\alpha 2$ helix, which is highlighted in orange. The orientation of the structures is identical to $\mathbf{b}$. $\mathbf{d}$ Ribbon representation of the structure of myosin VI bound to $\mathrm{CLCa}{ }^{46-61}$ using the same color scheme and orientation as in $\mathbf{c}$. e Ribbon representation as in $\mathbf{d}$, expanded and rotated about the CLCa helix to highlight the $\alpha$-helical structure of CLCa $46-54$ and interactions involving the Cterminal amino acids of the CLCa peptide. Sidechain heavy atoms of the highlighted residues are included. Note that the C-terminal portion of the CLCa fragment remains in close contact with myosin $\mathrm{VI}$ and the $\mathrm{CLCa}$ helix. $\mathbf{f}$ Selected regions from a $3 \mathrm{D}{ }^{13} \mathrm{C} \mathrm{NOESY}$ experiment acquired on $0.4 \mathrm{mM}{ }^{13} \mathrm{C}$, ${ }^{15} \mathrm{~N}-$ labeled CLCa 46-61 and equimolar unlabeled myosin VI1050-1131. NOE interactions involving the C-terminal portion of CLCa (A59, P60, and G61) are shown. Intramolecular and intermolecular interactions are labeled in red and black, respectively

\section{Table 1 NMR data collection and refinement statistics}

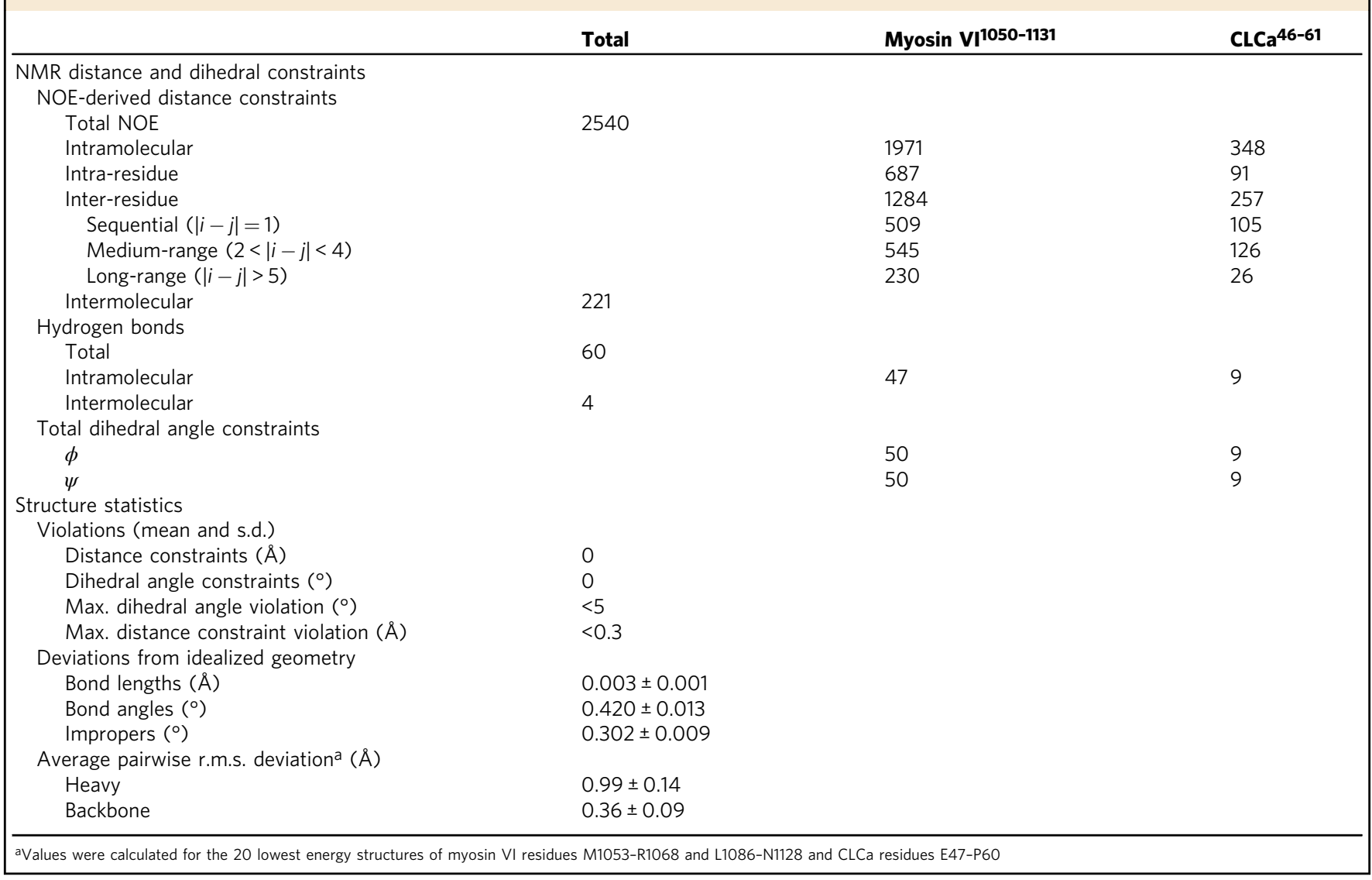

As predicted by the binding selectivity of the long isoform ${ }^{18}$, the interaction surface on myosin VI involves isoform-specific residues from a2, including P1055, M1058, A1059, and M1062, which surround L55 of CLCa (Fig. 4a, b). Myosin VI P1055 extends this CLCa-binding surface through its interaction with F52 (Fig. 4b, NOEs shown in Supplementary Fig. 7b). At an adjacent side of the CLCa helix, myosin VI R1117, V1120, Y1121, and W1124 surround CLCa A51 and I54 (Fig. 4a, b). In addition, the W1124 indole group forms a hydrogen bond to the sidechain carboxyl group of E50 (Fig. 4a).

The RRL motif required for binding to multiple adaptor proteins including optineurin and GIPC ${ }^{18,25,26}$ is embedded in $a 4$ (Supplementary Fig. 9a). R1116 does not participate in the interaction and remains surface exposed, whereas both R1117 and
L1118 contribute to CLCa binding. R1117, required for myosin VI structural integrity ${ }^{18,27}$, maintains its hydrogen bonds to S1087 and E1113 as in free myosin VI and forms a hydrogen bond to the backbone oxygen of CLCa D56, the sidechain of which also forms a hydrogen bond to the backbone amide of myosin VI Y1091 (Fig. 4c). Lastly, L1118 of the RRL motif contributes to binding though interactions with CLCa L55 (Fig. 4b). Notably, the CLCa amino acids critical for binding to myosin VI, including A51, I54, L55, and D56, are not conserved in CLCb (Fig. 4d), thus providing an explanation for paralog specificity.

The importance of the identified interactions is supported by GST pull-down experiments. A truncated construct confirmed that the $a 4$ helix of myosin VI is involved in binding to CLCa 
a

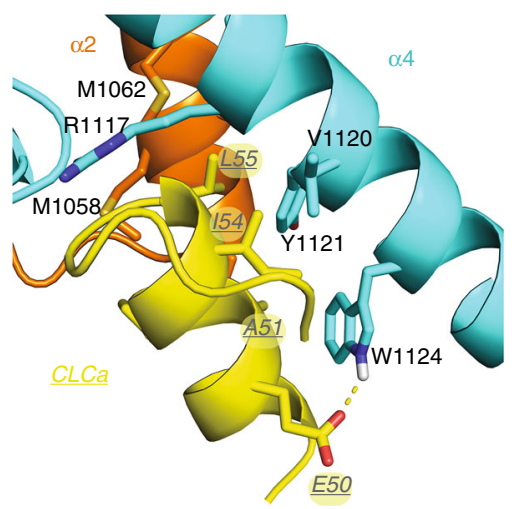

C

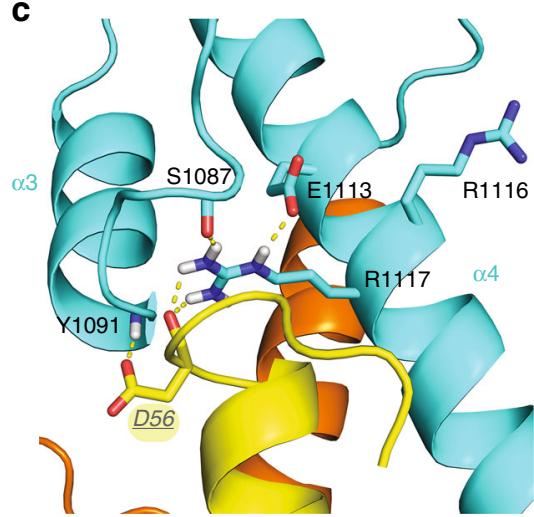

b

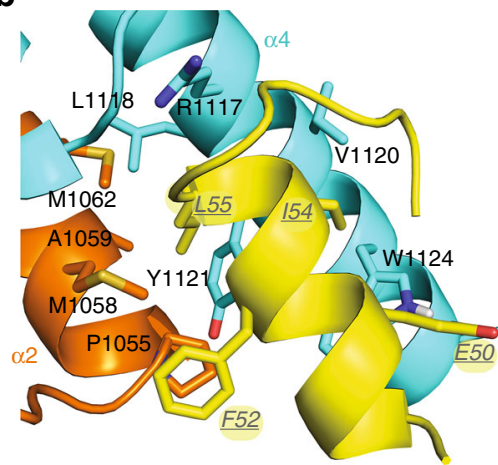

d

CLCa 46 IENDEAFAILDGGAPG 61 CLCb 38 IENDEGFGAPAGSHAA 53

f $* * * * * * * * *$

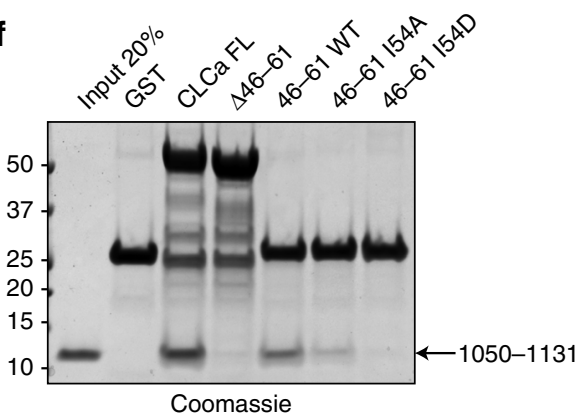

e GST-myosin VI ${ }^{1050-1131}$

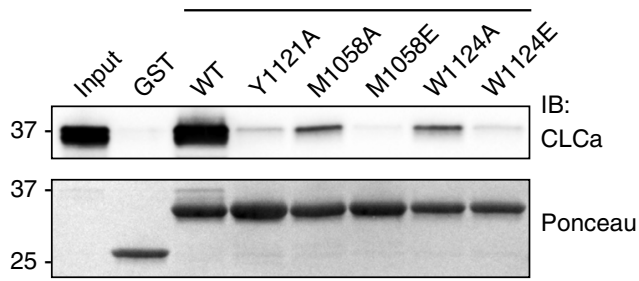

Fig. 4 A hydrophobic pocket formed by myosin VI encompasses residues 154 and L55 of CLCa. a Ribbon representation of the myosin VI:CLCa binding interface in the orientation of Fig. 3e with key sidechain heavy atoms displayed. Myosin VI $\alpha 4$ residues R1117, V1120, Y1121, and W1124 (blue) interact with CLCa E50, A51, 154, and L55 (yellow). CLCa L55 also interacts with myosin VI $\alpha 2$ residues, with M1058 and M1062 (orange) observable in this view. At the edge of the hydrophobic pocket lies a hydrogen bond between CLCa E50 and the myosin VI indole group. Sulfur and nitrogen atoms are in yellow and indigo, respectively. b As in a but rotated about the CLCa helix to highlight interactions involving the myosin VI isoform-specific $\alpha 2$ helix, especially P1055 and A1059. c Enlarged representation of the region containing myosin VI R1117 to highlight intramolecular and intermolecular hydrogen bonds. The guanidine group of R1117 forms hydrogen bonds to the backbone and sidechain carboxyl groups of myosin VI S1087 and E1113, respectively, and to the backbone carboxyl group of CLCa D56. This view is similar to that of $\mathbf{a}$ but rotated about the sidechain of R1117. In $\mathbf{a}$, c, a dashed yellow line is used to indicate a hydrogen bond with oxygen and nitrogen atoms in red and indigo, respectively. $\mathbf{d}$ Sequence alignment of CLCa 46-61 with the corresponding region of CLCb. Asterisks indicate residues not conserved between isoforms. In yellow are amino acids putatively responsible for the selective binding. e Pull-down assay using the indicated GST-myosin VI1050-1131 mutant constructs and lysates (1 mg) from HEK293T cells. After washes, bound proteins were eluted in Laemmli-buffer, resolved through SDS-PAGE, and IB was performed with the anti-CLCa antibody ( $\times 16)$. Ponceau detects equal loading of GST proteins. Representative image of three independent experiments is shown. $\mathbf{f}$ GST pull-down assay using the indicated CLCa constructs and purified fragment spanning amino acids 1050-1131 of myosin VI long. After washes, bound proteins were eluted in Laemmli-buffer, resolved through SDS-PAGE, and stained with Coomassie. Bottom panel, quantitation of three independent experiments. Data are expressed as percentage of binding with respect to input and normalized for the amount of GST proteins used in each pull-down. Error bars represent s.d. ${ }^{\star \star \star} P<0.001$ by two-tailed $T$ test

(Supplementary Fig. 9a) while single substitution of myosin VI M1058, Y1121, or W1124 led to reduced binding (Fig. 4e). FP analysis revealed a 2 log-fold difference in binding affinity for the Y1121A mutant (Supplementary Fig. 9b). On the CLCa side, we tested the effect of substituting I54 with alanine or aspartic acid, using CLCa full-length protein as a control. As expected, myosin $\mathrm{VI}^{1050-1131}$ bound to CLCa WT but not $\triangle 46-61$. $154 \mathrm{~A}$ or I54D significantly impairs binding to myosin VI, with aspartic acid showing the strongest defect (Fig. $4 \mathrm{f}$ ).

Myosin VI requirement for CME in polarized cysts. While CLCa is ubiquitously expressed in animal tissues ${ }^{5}$, the presence of myosin $\mathrm{VI}_{\text {long }}$ is restricted to organs containing polarized cells of 

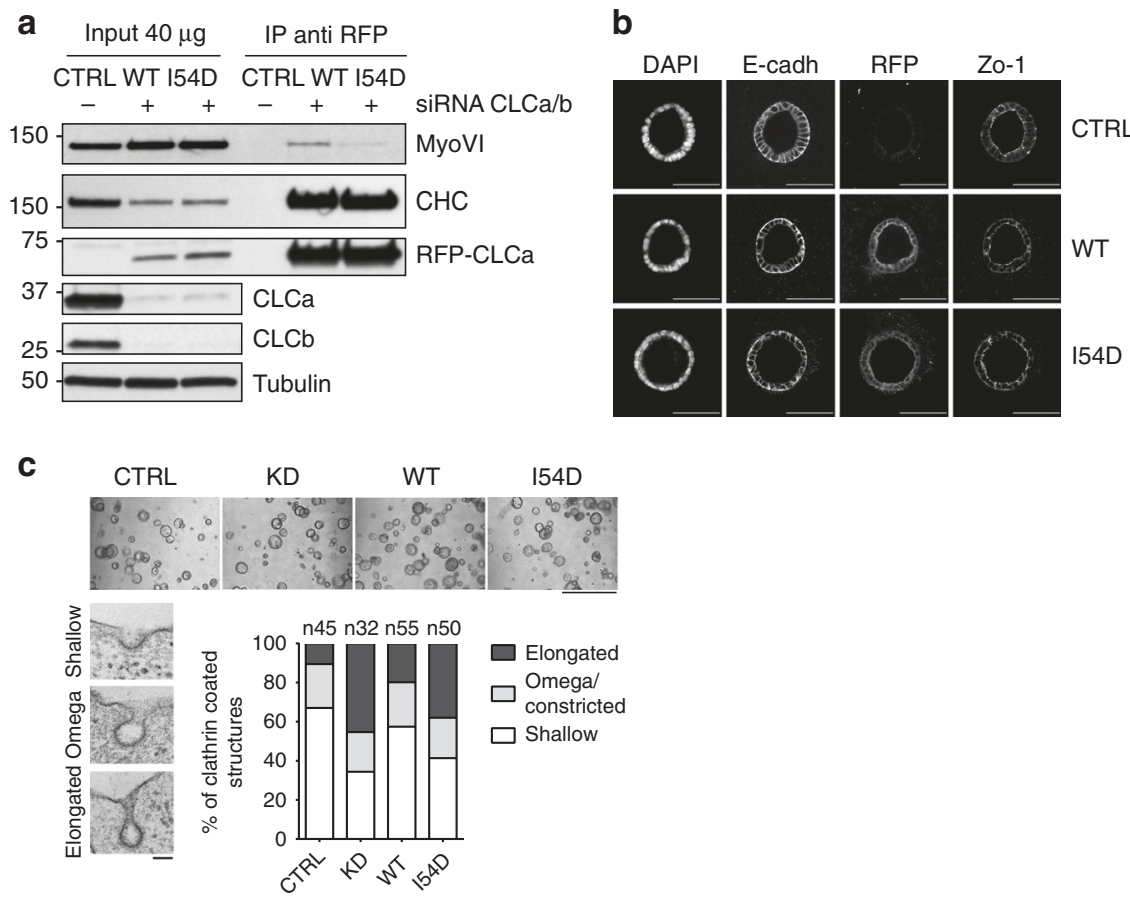

Fig. 5 CLCa I54D is a selective myosin VI-impaired mutant. a Co-immunoprecipitation experiment using lysates from Caco-2 reconstituted cell lines. Cells depleted of endogenous CLCs or mock treated are kept in confluency for 7 days, lysed, and RFP-CLCa wild-type and I54D were immunoprecipitated from $1 \mathrm{mg}$ of lysates. IP and IB as indicated. b Representative confocal micrographs of CLCa wild-type and I54D localization in Caco-2 cysts. Staining with the indicated apical-basal polarity markers are shown. Scale bar, $100 \mu \mathrm{m}$. c Upper panel, representative brightfield pictures of Caco-2 cysts generated using the indicated isogenic cell lines. Scale bar, $1 \mathrm{~mm}$. Clathrin-coated structures present at the apical surface of the Caco-2 cysts were counted and classified as shallow, omega/constricted, or elongated according to their morphology. Left panel, representative EM images of the different morphology of CCPs. Scale bar, $100 \mathrm{~nm}$. Right panel, distribution of clathrin-coated structures in the different isogenic cell lines expressed as percentage among the different classes and calculated on three independent experiments. Between 94 and 125 cellular profiles of well-polarized cells for each line were imaged. Number of clathrin-coated structures counted in total are reported as $n=x$. See also Supplementary Fig. 11d

epithelial origin, such as kidney and intestines, both in mice ${ }^{28}$ and humans (Supplementary Fig. 10a). There, myosin VI localizes to the apical surface facing the lumen of the organs at the base of microvilli ${ }^{29,30}$ (Supplementary Fig. 10b). To analyze the physiological role of the CLCa:myosin VI complex in a cellular model of polarized epithelial tissue, we took advantage of the intestinederived epithelial Caco-2 cells that form polarized cysts when plated as a single-cell suspension embedded in 3D EHS-derived matrix $^{31}$. Notably, in this Caco-2 cellular model system, a clear switch toward the myosin $\mathrm{VI}_{\text {long }}$ isoform occurs during the acquisition of full polarity both in $2 \mathrm{D}$ and $3 \mathrm{D}$ systems, as measured by reverse transcriptase-polymerase chain reaction (PCR) (Supplementary Fig. 10c). Transmission electron microscopy (TEM) and confocal microscopy analysis showed that the cysts are fully formed and polarized (Supplementary Fig. 10d-f) and myosin $\mathrm{VI}_{\text {long }}$ is enriched in the apical terminal web region together with occludin (Supplementary Fig. 10d). We then generated Caco-2 cells stably expressing red fluorescent protein (RFP)-WT or an RFP-I54D mutant rat CLCa as these constructs are resistant to the small interfering RNA (siRNA) oligos designed on the human sequence. Upon efficient depletion of the endogenous CLCa and CLCb by siRNA oligos (Supplementary Fig. $11 \mathrm{a}-\mathrm{c}$ and Fig. 5a), co-immunoprecipitation analysis performed with lysates from $2 \mathrm{D}$ fully polarized Caco-2 cells demonstrated that the I54D mutant was largely unable to interact with myosin VI (Fig. 5a), validating our previous in vitro results. Next, single Caco-2 reconstituted cells depleted of endogenous CLCs were cultured in matrigel and 7 days after cysts were counted and stained to evaluate adherens and tight junctions and the localization of RFP-CLCa WT or I54D mutant (Fig. 5b).
Exogenous RFP-CLCa I54D mutant, similarly to the WT protein, was enriched in the apical region toward the lumen and did not appear to affect the ability of the cells to form cysts (Fig. 5c) or prevent the establishment of apical basal polarity (Fig. $5 \mathrm{~b}$ and Supplementary Fig. 10e, f).

Clues as to the molecular requirement for CLCa-myosin VI interaction were derived from EM analysis of the cysts. As CLCs are implicated in CME, we investigated the CCPs that formed at the apical surface facing the lumen in control, CLC-depleted, and reconstituted RFP-CLCa variants. CCPs stemming from the PM were classified as shallow, omega/constricted, or elongated according to their morphology (Fig. 5c, left panel). Compared to mock-treated Caco-2 cysts, cysts depleted of CLCs displayed a significantly greater proportion of CCPs with an aberrant morphology, consisting of elongated pits joined to the PM by "long necks" (Fig. 5c). These structures closely resembled those induced by the dominant-negative mutant of dynamin, DynK $44 \mathrm{~A}^{32}$. Strikingly, while WT RFP-CLCa fully rescued this phenotype, the I54D mutant was unable to do the same, indicating that the morphological defect scored in the absence of CLCs can be ascribed to the lack of myosin VI interaction with CLCa (Fig. 5c, Supplementary Fig. 11d).

These results are compatible with the possibility that the interaction of CLCa with myosin VI is part of the machinery that controls CCP fission. Previously, CLCs and their binding partner Hip1R were implicated in this process at the apical membrane of polarized cells ${ }^{6}$. Intriguingly, the myosin VI-binding region we identified here on CLCa partially overlaps with the Hip1Rbinding region ${ }^{7}$ (Fig. 6a). Thus we evaluated the ability of myosin VI and Hip1R to bind simultaneously to CLCa, using an in vitro 
a

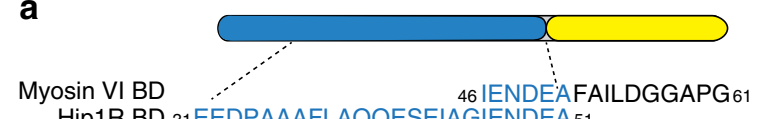
Hip1R BD 31 EEDPAAAFLAQQESEIAGIENDEA 51

b

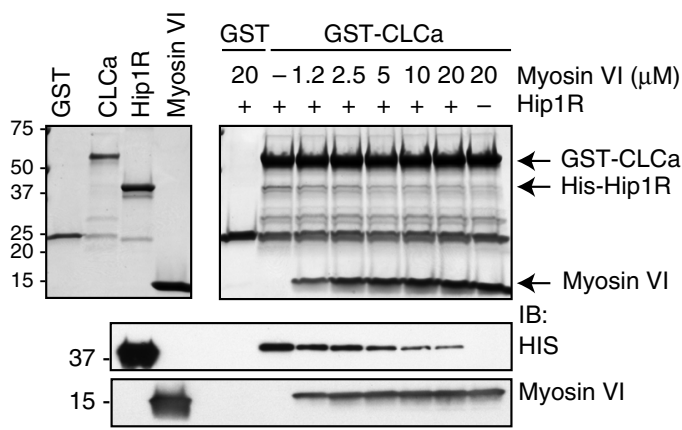

C

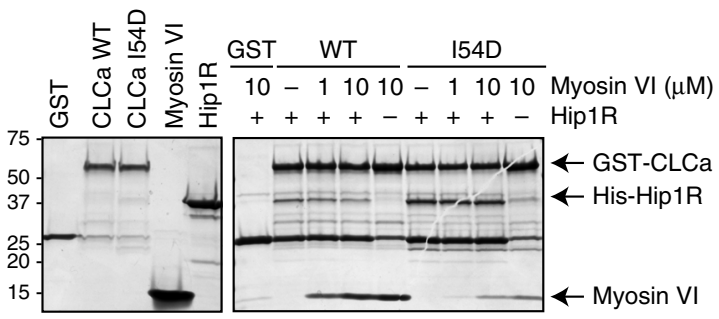

d
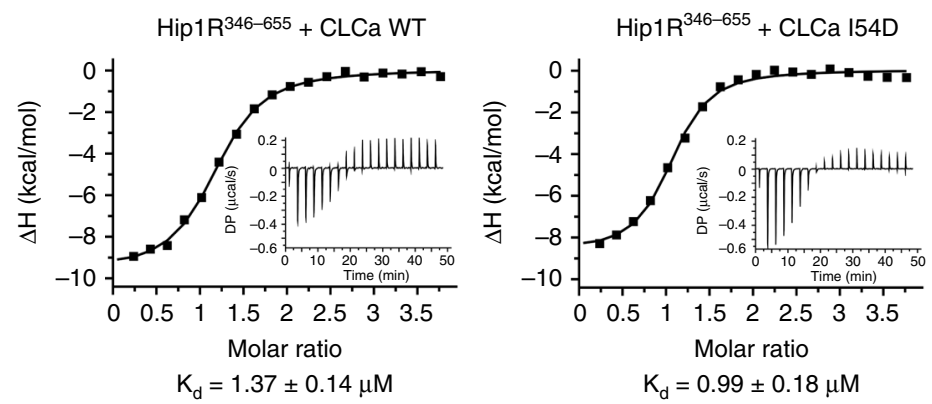

e

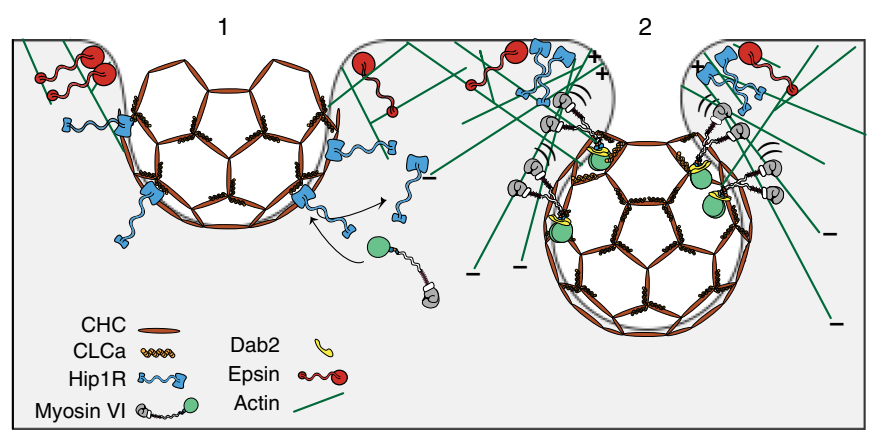

Fig. 6 Myosin VI and Hip1R are mutually exclusive binders of CLCa. a Binding regions of myosin VI and Hip1R on CLCa. b Competitive binding assay. Bacterially purified His-Hip1R coiled-coil region spanning residues 346-655 (2.5 $\mu \mathrm{M})$ pre-incubated with GST-CLCa full-length protein $(1 \mu \mathrm{M})$ for $1 \mathrm{~h}$ at $4{ }^{\circ} \mathrm{C}$ was mixed with increasing amounts of myosin V11050-1131 as indicated. Bottom panel, Coomassie staining. Lower panels, 1/10 of the elutant was loaded for immunoblotting with the indicated antibodies. $\mathbf{c}$ As in $\mathbf{b}$ but using GST-CLCa I54D mutant. d ITC experiments with the indicated proteins. The integrated heat and raw plots are reported. Equilibrium dissociation constants $\left(K_{d}\right)$ obtained by the fitting are indicated below. Relevant ITC measurements are reported in Table 2. e A putative model of clathrin-coated pit fission in polarized tissue. Once CCP passes the endocytic checkpoint, it undergoes a maturation process mediated by PIP2 turnover and the activity of several endocytic accessory factors (e.g., Hip1R). (1) As the bud expands, myosin VI is recruited to CCPs by CLCa and disengages Hip1R, which preferentially associates with epsin at the CCP edge. Actin anchoring and polymerization is thereby restricted to the neck of the invaginating pit where Hip1R along with epsin provide a link between actin nucleation and the CCP. Alternatively, formation of a complex between epsins and Hip1R at the neck would recruit Hip1R from the coat, exposing the myosin VI-binding site on CLCa and causing recruitment of myosin VI to the CCP. (2) Myosin monomer bound to CLCa anchors the CCP to the actin meshwork engaged in retrograde flow and facilitates movement of the vesicle into the cytoplasm. After dimerization induced by Dab2 or oligomerization, myosin VI becomes a processive motor that walk toward the minus end of actin filaments providing mechanical force to oppose membrane tension in polarized tissues and promoting the final dynamin-mediated fission step (not depicted). Future experiments are needed to test this model. In the myosin VI representation, the boxing glove and white cuff indicate the motor domain and single IQ motif, respectively, while the cargo-binding tail, which includes the clathrin-binding domain, is depicted as a green sphere; the black squiggly connecting region includes the $3 \mathrm{HB}$ and $\mathrm{SAH}$

Table 2 Thermodynamic parameters obtained by isothermal titration calorimetry

\begin{tabular}{lllllll} 
& $\boldsymbol{N}(\mathbf{s i t e s})$ & $\boldsymbol{\Delta H}(\mathbf{k c a l} / \mathbf{m o l})$ & Offset (kcal/mol) & $\boldsymbol{\Delta G}(\mathbf{k c a l} / \mathbf{m o l})$ & $-\boldsymbol{T} \Delta \boldsymbol{S}(\mathbf{k c a l} / \mathbf{m o l})$ & $\boldsymbol{K} \mathbf{d}(\boldsymbol{\mu M})$ \\
\hline Hip1R + CLCa FL & $1.15+0.01$ & $-9.70+0.19$ & $2.27+0.06$ & -8.00 & 1.69 & $1.37+0.14$ \\
Hip1R + CLCa I54D & $1.01+0.02$ & $-8.72+0.27$ & $1.25+0.08$ & -8.19 & 0.528 & $0.99+0.18$ \\
My06 + CLCa FL & 0.79+0.02 & $-11.6+0.47$ & $2.02+0.18$ & -9.00 & 1.69 & $0.09+0.05$ \\
Myo6 + CLCa I54D & N/A & N/A & N/A & N/A & N/A & N/A \\
\hline
\end{tabular}

N/A not applicable

assay with bacterially purified components. We incubated GSTtagged CLCa full-length protein with the coiled-coil region of Hip1R spanning amino acids 346-655 and added increasing amounts of myosin VI ${ }^{1050-1131}$ protein fragment. Analysis by Coomassie staining and immunoblotting showed that myosin VI addition caused progressive displacement of Hip1R from CLCa (Fig. 6b), indicating a direct competition between the two CLCa interactors possibly due to steric hindrance. Consistent with separate but proximal binding sites for these two CLCa-binding proteins, Hip1R bound both the CLCa I54D mutant and WT CLCa, but its binding to the mutant was not affected by myosin VI (Fig. 6c). Notably, CLCb interaction with Hip1R was insensitive to the addition of myosin VI in the same competition assay (Supplementary Fig. 11e), confirming once again the selectivity of myosin VI for CLCa. Finally, we measured the affinity of the two interactors for CLCa full length by isothermal calorimetric assay (isothermal titration calorimetry (ITC), Table 2). Myosin VI bound CLCa with a $K_{\mathrm{d}}$ of $90 \mathrm{nM}$, while it 
showed no binding to CLCa I54D (Supplementary Fig. 11f). In agreement with the competition assay, Hip1R binds WT and mutant CLCa equally well but with a $K_{\mathrm{d}}$ a $\log$ higher with respect to myosin VI (Fig. 6d).

Altogether, these data provide evidence for coordinated action of myosin VI and CLCa in the fission of CCPs at the apical surface of polarized cells and identify a direct competition between Hip1R and myosin VI.

\section{Discussion}

It is well established that actin remodeling during CME is required when the PM tension is high ${ }^{6}$ or when there is a need to change the mechanical properties of the clathrin lattice to engulf non-canonical bulky cargo, such as viral or bacterial pathogens ${ }^{10,12}$. The functional link between CCPs and actin is represented by the CLC-Hip1R interaction in both yeast and mammals ${ }^{33-35}$. Here we identified the role of a third player in this process, myosin VI, which selectively binds to CLCa and directly competes with Hip1R. While myosin VI has been previously implicated in $\mathrm{CME}^{26,36,37}$, our structural definition of the CLCa-myosin VI interaction provides the molecular details of such involvement.

Myosin VI is unique among mammalian myosins in that it moves toward the minus end of F-actin ${ }^{38}$. This unique motor protein supports critical functions at multiple cellular sites via interaction with different adaptors that target it to specific organelles. We recently showed that an alternatively spliced a2linker modulates the myosin VI interactome and is the causative link to its diverse functions ${ }^{18}$. In particular, the myosin $\mathrm{VI}_{\text {long }}$ isoform includes the exon cassette that codes for the a2-linker and is endowed with clathrin-binding capacities and endocytic functions ${ }^{18}$.

Here we reveal that myosin VI is recruited to CCPs via a selective interaction with CLCa. Our biochemical and structural characterization of the myosin VI-CLCa interaction identified contacts unique to CLCa that are contributed largely from a helical structure. Key differences in the amino acid sequence of the CLCa and CLCb paralogs are likely responsible for the lack of binding between myosin VI and CLCb (Fig. 4d). Specifically, A51 and I54 in CLCa are both replaced with smaller residues (Gly and Ala, respectively, in CLCb), which likely diminishes the hydrophobic contacts with myosin VI (Fig. 4a, b). In CLCb, D56 is replaced with an alanine, which cannot form a hydrogen bond with Y1091 in myosin VI (Fig. 4c). Finally, L55, which embeds itself between helices $a 2$ and $a 4$ of myosin VI (Fig. $4 a, b$ ), is replaced with a proline in $\mathrm{CLCb}$. Owing to the structural rigidity of proline, this amino acid may disrupt the helical turn, possibly preventing $\mathrm{CLCb}$ from fitting into the myosin VI-binding pocket that accommodates CLCa.

The CLCa peptide identified here as determinant for myosin VI interaction was invisible in the previously published structures of the clathrin lattice and the central domain of the triskelion ${ }^{8,23}$ and identified as a conformationally labile peptide modulated by calcium ion concentration ${ }^{39}$, strongly suggesting that this flexible peptide is an important element of regulation for CLC. Calcium was also shown to mobilize myosin VI from a compact dormant state into a cargo-binding primed state ${ }^{40}$. Interestingly, the segment of the myosin VI tail region mediating this regulation (amino acids 1060-1125) matches exactly with the clathrinbinding domain. In resting conditions, myosin VI adopts a backfolded, dormant state, with the tail domain amino acids 1060-1125 binding to apo-calmodulin; at high calcium levels, myosin VI unfolds and the release of the myosin VI tail mobilizes the motor, priming it to bind to cargo ${ }^{40}$. In this open structural conformation of myosin VI, CLCa binding should be more favorable. Consistently, addition of $2 \mathrm{mM} \mathrm{Ca}^{2+}$ to the lysis buffer significantly improved the co-immunoprecipitation between myosin VI and CLCa (Supplementary Fig. 12). Thus our results, together with these previous findings, suggest that release of intracellular calcium could orchestrate the timing of localized myosin VI activation and of clathrin-myosin VI functional interaction.

Like Hip $1 \mathrm{R}^{41}$ and $\mathrm{CLC}^{42}$, myosin VI is largely dispensable for $\mathrm{CME}$ in $2 \mathrm{D}$ culture in vitro. However, the specific expression of myosin $\mathrm{VI}_{\text {long }}$ in polarized tissues (e.g., kidney and intestine), together with its features as an actin-based motor, prompted us to examine its function in Caco- 2 cysts, used as proxy of the enterocytes lining the small intestine.

We show here that, in Caco-2 grown as cysts, a clear switch toward myosin $\mathrm{VI}_{\text {long }}$ occurs as cysts develop with this endogenous isoform enriched at the apical membrane, together with clathrin-rich structures. As further discussed below, CME in epithelial polarized cells is dependent on actin polymerization, which drives membrane invagination against the membrane tension that rigidifies the apical membrane ${ }^{6}$. In this process, CLCa may recruit myosin VI to CCPs to provide the force necessary to complete neck constriction, fission, and release. Indeed, using a genetic reconstitution approach, we were able to demonstrate a clear change in the distribution of CCP structures at the apical surface when we abrogated the CLCa-myosin VI interaction. The increased number of elongated CCPs present at the apical surface of CLC-depleted cysts or cysts reconstituted with I54D CLCa mutant, which does not bind myosin VI, suggests a delay in CCP progression at this surface. Our results are compatible with the idea of myosin VI acting at the neck of a mature CCP to promote fission. Consistent with this notion, recent evidence demonstrated that myosin VI, together with branched actin filaments, mediates severing by constricting the neck of budding tubules that are released from melanosomes ${ }^{43}$.

The involvement of the myosin VI-CLCa interaction in the CCP fission step at the apical membrane of polarized cells is attractive, as this step is where actin has also been implicated in yeast ${ }^{44}$ and observed in mammalian cells ${ }^{45}$. It is tempting to speculate that the lack of CLCa-myosin VI interaction and thus a defective CME is the cause of the altered level of CTFR, megalin receptor and sodium type-IIa cotransporter $(\mathrm{NaPi} 2 \mathrm{a})$ observed at the apical membrane of myosin VI-depleted enterocytes and renal proximal tubular cells ${ }^{19,46-51}$.

Our results unveil a direct competition between myosin VI and Hip1R for CLCa (Fig. 6a-d) that likely establishes partitioning of actin activity during the various stages of CCP formation (Fig. 6e). Current knowledge localizes Hip1R bound to CLCs throughout the CCP as well as inserted directly in the surrounding PM via its lipid-binding ANTH domain ${ }^{52,53}$. The conformational change induced by CLC binding masks the actin-binding domain of Hip proteins in the coat ${ }^{8}$ and would exclude actin interaction at this site. At the adjacent membrane, Hip protein interaction with the epsin family proteins, both in yeast ${ }^{52}$ and in mammals ${ }^{54}$, results in a multimeric heterocomplex ${ }^{52,55}$ that may preferentially occur at the neck of an invaginating $\mathrm{CCP}^{56}$. Here Hip proteins dissociated from the clathrin coat could interact with actin, generating the active site required for the invagination of coated pits. Indeed, depletion of either Hip1R or epsins results in similar abnormal actin nucleation at endocytic sites where vesicle budding is inhibited $^{33,54}$. Thus regulation of actin activity by Hip proteins seems to be restricted to the neck of an invaginating vesicle ${ }^{8}$ where barbed ends of branched actin filaments are concentrated ${ }^{45}$ and fission occurs as dynamin assembles into a helical coat ${ }^{57,58}$. How might this happen? We propose here that myosin VI contributes to the temporal regulation of Hip1R action while producing the forces to overcome high membrane tension (Fig. 6e). During 
vesicle invagination, myosin VI recruited by CLCa would compete out Hip1R, promoting its dissociation from the cages and recruitment by epsins at the uncoated neck. At the vesicle neck, Hip1R regulates actin filament polymerization ${ }^{33}$ while Dab2 may tether myosin VI recruited by CLCa into a stable and active dimer ${ }^{59}$. Finally, acting as a processive cellular motor on branched actin filaments, myosin VI may contribute to the release of the invaginating vesicle, promoting CCP fission (Fig. 6e). Notably, our findings are in line with previous studies based on total internal reflection fluorescence microscopy that revealed myosin VI to be recruited during a late stage of clathrin-mediated internalization, concomitantly with dynamin and regulators of actin polymerization, to promote the scission of the invaginated membrane ${ }^{60}$. Consistent with the idea of myosin VI actively participating in the latest steps of CCP maturation, in vivo analysis of renal proximal tubule cells in mice lacking myosin VI showed reduced uptake of endocytic markers and accumulation of AP2 and Dab2 at the $\mathrm{PM}^{49}$.

Our findings indicate that only CLCa-containing CCPs may be subjected to the regulation exerted by myosin VI. CLCa-null mice lack the myosin VI-CLCa interaction and its unique function and this deficiency may contribute to the $50 \%$ postnatal mortality rate of these animals ${ }^{5}$. Consistent with this idea, CLCa is ubiquitously expressed at high levels in all vertebrate tissues, whereas CLCb characteristically exhibits variable levels ${ }^{5}$, and compared to $\mathrm{CLCb}$, CLCa is preferentially associated with peripheral clathrin-coated structures and proteins involved in cell spreading ${ }^{15}$, a process that also requires actin regulation.

We are at an early stage of appreciating the extent of the selective activity of each CLC paralog, with hints of CLCa- and $\mathrm{CLCb}$-associated functions emerging in recent reports ${ }^{15-17}$. The high conservation and distinction of the two CLC-encoding genes that are preserved within all vertebrates ${ }^{61}$ strongly support the notion that CLCa and CLCb proteins have non-redundant functions. In the emerging scenario, our data indicate CLCa as a key modulator of clathrin interactions with the actin cytoskeleton.

\section{Methods}

Cell lines and KO mouse strains and constructs. HEK293T cells (ICLC) were grown in Dulbecco's Modified Eagle Medium (DMEM) supplemented with 10\% fetal bovine serum (FBS) S.A. and $2 \mathrm{mM} \mathrm{L-glutamine.} \mathrm{HeLa} \mathrm{cells} \mathrm{(ATCC)} \mathrm{were}$ grown in Minimum Essential Medium (MEM) supplemented with 10\% FBS S.A., $0.1 \mathrm{mM}$ non-essential amino acids, $2 \mathrm{mM}$ L-glutamine, and $1 \mathrm{mM}$ Na-Pyruvate. CaCo-2 cells (ATCC) were grown in MEM supplemented with $20 \%$ FBS S.A., 0.1 $\mathrm{mM}$ non-essential amino acids, and $2 \mathrm{mM}$ L-glutamine.

At each batch of freezing, all cell lines were authenticated by STR profiling (StemElite ID System, Promega) and tested for mycoplasma using PCR and biochemical test (MycoAlert, Lonza).

Caco-2 RFP-CLCa WT and CLCa I54D isogenic cell lines were generated by lentiviral infection of Caco-2 cells with the corresponding pLVX-RFP expressing rat-CLCa variants and bulk populations were FACS-sorted to enrich for RFPpositive cells.

Production of mice deleted for the CLTA gene encoding CLCa (CLCa KO) was previously described ${ }^{5}$. Mice deleted for the CLTB gene (CLCb KO) were generated in the Brodsky laboratory and loss of CLCb in the tissues studied here has been confirmed, as verified in Supplementary Fig. 2a.

Human constructs of myosin VI were previously described ${ }^{18,27}$. Mutants generated here were engineered by site-directed mutagenesis and sequence verified. Details are available in Supplementary Table 1.

pRSF-duet codifying for His-Hip1R coiled coil region (amino acids 346-655) was previously described ${ }^{8}$.

pGEX6P1-CLCa FL construct was generated by subcloning RFP-hCLCa, kindly provided by Klemens Rottner (HZI, Braunschweig), into pGEX6P1 with XhoI enzyme. Subsequent truncated constructs were engineered by site-directed mutagenesis or recombinant PCR and sequence verified. Details are available upon request.

pGEX6P1-CLCb FL construct was generated by PCR using as template pCMV tag 2B-hCLCb, kindly provided by Elizabeth Smythe (University of Sheffield), and sequence verified.

pLVX lentiviral vector expressing rat RFP-CLCa was cloned from pMSCV-RFPratCLCa vector, kindly provided by Tom Kirchhausen (Harvard Medical School, Boston), using the InFusion system (Takara Clontech) and the following oligos: forward: CTCAAGCTTCGAATTCATGGTGTCTAAGGGCGAAGAGCTG reverse: GTCGACTGCAGAATTCTCAATGCACCAGGGGCGC.

pLVX RFP-CLCa I54D mutation was generated by site-directed mutagenesis and sequence verified.

pEGFP-CHC and pGEX6P1-CHC constructs described in Supplementary Fig. 1a were kindly provided by Stephen Royle (University of Liverpool).

5-Carboxyfluorescein (5-FAM)-conjugated CLCa peptides 46-61, 51-61 and 51-66 were synthesized by GenScript.

Antibodies. All antibodies used in this study are listed in Supplementary Table 2.

CLC depletion. Transient CLCa and/or CLCb KD were performed using Stealth siRNA oligonucleotides from ThermoFisher Scientific (humanCLCA-3utr target sequence: 5'-TGGAAACACTACATCTGCAATATCT-3' and humanCLCB-3utr target sequence: 5'-CGCCTCCTCTCAGTCTACTCAATTG-3') or Qiagen [humanCLCa (2) target sequence: AGACAGTTATGCAGCTATT].

Caco-2 (or HeLa, Supplementary Figs. 1 and 11d) cells were transfected using RNAiMax (Invitrogen) and siRNA oligos at $8 \mathrm{nM}$ final concentration. Cells were transfected twice, first in suspension and the day after in adhesion. The third day Caco- 2 cells reach confluency and were kept confluent for additional 7 days in order to obtain a compact polarized monolayer. Medium was changed every 2 days.

Caco-2 cyst formation. Caco-2, Caco-2 RFP-rCLCa WT, and Caco-2 RFP-rCLCa I54D cell lines were transiently transfected with CLC oligos. Two days after siRNA transfection, single-cell suspensions were embedded in medium plus 50\% Matrigel (Corning) and $1 \mathrm{mg} / \mathrm{ml}$ collagen mixture and plated on 8-well ibidi chamber (Cat. No: 80826) at a density of $9 \times 10^{3}$ cells/well. Approximately $150 \mu \mathrm{l}$ were plated in each well, allowed to solidify for $30 \mathrm{~min}$ at $37^{\circ} \mathrm{C}$, and overlayed with $200 \mu \mathrm{l}$ of medium. Medium was changed every 2 days. At day 6, cholera toxin (Sigma Cat. No.: C8052) was added at a concentration of $0.1 \mu \mathrm{g} / \mathrm{ml}$ and cysts are collected the day after.

Immunofluorescence (IF) and co-localization experiments. For IF analysis, samples were fixed with $4 \%$ paraformaldehyde for $10 \mathrm{~min}$ and permeabilized at room temperature (RT) with $0.5 \%$ Triton-X100 in PBS for $10 \mathrm{~min}$ (cells) or $20 \mathrm{~min}$ (mice tissue). Blocking was performed in phosphate-buffered saline (PBS) containing $2 \%$ bovine serum albumin (BSA) for $1 \mathrm{~h}$ prior to the incubation of the primary antibodies in PBS containing $0.1 \%$ Triton X-100 and $2 \%$ BSA overnight at $4{ }^{\circ} \mathrm{C}$. After extensive washing, sample were incubated with secondary antibodies $45 \mathrm{~min}$, at RT. Coverslips were mounted in a glycerol solution ( $20 \%$ glycerol, 50 $\mathrm{mM}$ Tris $\mathrm{pH}=8.4$ ) to avoid mechanical deformation of the sample. Images were captured using a HCX $\times 63$ plan-apochromat objective on a Leica inverted SP5 or SP8 microscope with a laser scanning confocal system. Analysis was performed with ImageJ (http://imagej.nih.gov/ij/).

For co-localization experiments in HeLa cells, to remove soluble cellular proteins, cells were extracted with $0.03 \%$ saponin in cytosolic buffer $(25 \mathrm{mM}$ Hepes-KOH, pH 7.4, $25 \mathrm{mM} \mathrm{KCl}, 2.5 \mathrm{mM}$ magnesium acetate, $5 \mathrm{mM}$ ethylene glycol-bis( $\beta$-aminoethyl ether)-N,N,N $\mathrm{N}^{\prime}$-tetraacetic acid (EGTA), $150 \mathrm{mM} \mathrm{K}$ glutamate) for $1 \mathrm{~min}$ prior to fixation. For co-localization analysis, regions of interest were drawn around individual cells. The Manders' coefficient was obtained using JACoP plugin and processed for statistical analysis with Prism (GraphPad software). Statistical significance was determined by non-parametric two-tailed $t$ tests. Sample size was chosen arbitrary with no inclusion and exclusion criteria

For IF analysis of Caco-2 cysts, cysts grown in matrigel in ibidi chambers were fixed in paraformaldehyde (PFA) $4 \%$ for $25 \mathrm{~min}$, washed three times in PBS-glycine $0.75 \%$, once in PBS, and permeabilized with $0.5 \%$ Triton X-100 for 20 min. Blocking was performed with $10 \%$ goat serum in IF buffer $(0.1 \%$ BSA, $0.05 \%$ Tween-20, $0.2 \%$ Triton X-100, $0.05 \% \mathrm{NaN}_{3}$ in PBS). Samples were incubated overnight in appropriate primary antibodies dilutions in IF buffer at RT, then washed three times in IF buffer and incubated at RT for $1 \mathrm{~h}$ with secondary antibody dilutions 1:100 in IF buffer and $15 \mathrm{~min}$ with 4',6-diamidino-2phenylindole. Finally, samples were washed in PBS and post-fixed in PFA 4\% for $10 \mathrm{~min}$. Images were captured using a Leica inverted SP2 microscope with a laser scanning confocal system.

Isoform detection by PCR. Expression of myosin VI isoforms in cell lines or tissues was assessed by PCR. Messenger RNA from cultured cells or cysts was isolated with Maxwell RSC instrument (Promega) according to the manufacturer's protocols. RNA from mouse kidney and intestine tissues was obtained from C57BL/6 WT mouse and from commercially available human RNA (Takara Clontech Cat.No.: 636539 and 636529 for intestine and kidney, respectively). Retro-transcription was performed with the High Capacity cDNA Reverse Transcription Kit (Applied Biosystem). cDNA obtained was used in PCR reactions with primers flanking the spliced region as described in ref. ${ }^{18}$.

Protein expression and purification. GST fusion proteins were expressed in Escherichia coli Rosetta (DE3) cells (Novagen) at $30^{\circ} \mathrm{C}$ for $4 \mathrm{~h}$ after induction with $0.8 \mathrm{mM}$ IPTG at an $\mathrm{OD}_{600}$ of 0.6 . Cell pellets were resuspended in lysis buffer 
(50 mM Na-HEPES pH 7.5, $150 \mathrm{mM} \mathrm{NaCl}, 1 \mathrm{mM}$ ethylenediaminetetraacetic acid (EDTA), 5\% Glycerol, 0.1\% NP40, Protease Inhibitor Cocktail set III, Calbiochem). Sonicated lysates were cleared by centrifugation at $48,000 \times g$ for $30 \mathrm{~min}$. Supernatants were incubated with $1 \mathrm{ml}$ of glutathione-sepharose beads (GE Healthcare) per liter of bacterial culture. After $4 \mathrm{~h}$ at $4{ }^{\circ} \mathrm{C}$, the beads were extensively washed with PBS/0.1\% Triton and equilibrated in storage buffer (50 mM Tris pH 7.4, 100 $\mathrm{mM} \mathrm{NaCl}, 1 \mathrm{mM}$ EDTA, $1 \mathrm{mM}$ DTT, 10\% glycerol). When needed, proteins were incubated overnight at $4{ }^{\circ} \mathrm{C}$ with PreScission Protease to obtain samples cleavaged from GST-tag. Cleaved proteins were further purified through Ion-Exchange chromatography on an ÄKTA Purifier FPLC system (GE Healthcare) and dialyzed overnight against storage buffer prior to snap-freezing in liquid nitrogen.

Biochemical experiments. For pull-down experiments, $1.5 \mu \mathrm{M}$ of GST-fusion proteins immobilized onto GSH beads were incubated for $1 \mathrm{~h}$ at $4{ }^{\circ} \mathrm{C}$ in JS buffer (50 mM Hepes pH 7.5, $50 \mathrm{mM} \mathrm{NaCl}, 1.5 \mathrm{mM} \mathrm{MgCl}_{2}, 5 \mathrm{mM}$ EGTA, $5 \%$ glycerol, and $1 \%$ Triton X-100) with either $1 \mathrm{mg}$ lysate produced in JC buffer or the indicated concentration of cleaved and purified proteins. After extensive washes with JS buffer, beads were re-suspended in Laemmli-buffer and proteins analyzed through SDS-PAGE. Detection was performed either by staining the gels with Coomassie or by immunoblotting using specific antibodies. Ponceau-stained membrane was used to show loading of GST-fusion proteins.

For competition experiments, $1.5 \mu \mathrm{M}$ of GST-CLCa immobilized onto GSH beads were incubated with $2.5 \mu \mathrm{M}$ of His-Hip1R ${ }^{346-655}$ for 1 hour at $4^{\circ} \mathrm{C}$ in JS buffer, then increasing concentration (from 1.2 to $20 \mu \mathrm{M}$ ) of myosin $\mathrm{VI}^{998-1131}$ were added and incubated for additional $30 \mathrm{~min}$ at $4^{\circ} \mathrm{C}$. Washes and detection as noted above.

For co-IP experiments, Caco-2 RFP-rat CLCa WT and I54D depleted of the endogenous CLCs as previously described, were lysed in JS buffer and IP was performed incubating $1 \mathrm{mg}$ of lysate with RFP-Trap (Chromotek) for $120 \mathrm{~min}$ at $4{ }^{\circ} \mathrm{C}$. After extensive washes with JS buffer, beads were re-suspended in Laemmlibuffer and proteins analyzed through sodium dodecyl sulfate-polyacrylamide gel electrophoresis (SDS-PAGE) and immunoblotting.

FP assays were carried out at $22^{\circ} \mathrm{C}$ on a 384 -well plate with Infinite 200 instrument (Tecan) using an excitation wavelength of $535 \mathrm{~nm}$ and an emission wavelength of $580 \mathrm{~nm}$. Concentrations in the nanomolar range $(20-50 \mathrm{nM})$ of 5FAM-conjugated CLCa peptides (GenScript) were titrated with the indicated myosin VI-purified fragment starting from an initial concentration of $\sim 150 \mu \mathrm{M}$. Polarization readings from three independent experiments were averaged and fitted as described in Eletr et al. ${ }^{62}$

For analytical size exclusion chromatography, $50 \mu \mathrm{M} \mathrm{CLCa}{ }^{46-61}$ was incubated at 1:1 molar ratio with $50 \mu \mathrm{M}$ of myosin $\mathrm{VI}^{998-1131}$ fragment for $15 \mathrm{~min}$ at $4{ }^{\circ} \mathrm{C}$ and subjected to size exclusion chromatography on a Superdex75 (5/150) column using the ÄKTA microsystem (GE Healthcare). Fractions containing complex or single proteins were analyzed by SDS-PAGE and Coomassie staining.

ITC measurements were performed in a buffer containing $20 \mathrm{mM}$ Tris- $\mathrm{HCl} \mathrm{pH}$ $8.0,150 \mathrm{mM} \mathrm{NaCl}$, and $2.5 \%$ glycerol, at $25^{\circ} \mathrm{C}$ on a MicroCal PEAQ-ITC (Malvern Panalytical, Malvern, UK) instrument. All protein samples were dialyzed overnight in this buffer prior to titration. In all, $380 \mu \mathrm{M}$ CLCa WT or I54D mutant were titrated into $20 \mu \mathrm{M}$ of either Hip1R $\mathrm{R}^{346-655}$ or myosin $\mathrm{VI}^{1050-1131}$ provided in the reaction cell. For each experiment, we used 19 injections of $2 \mu \mathrm{l}$ with $150 \mathrm{~s}$ spacing and $750 \mathrm{rpm}$ stirring speed. Measurements were performed in duplicates or triplicates. Raw data were analyzed with the integrated Malvern analysis software, and heat production was fitted to a one-set-of-sites binding model.

CCV preparation from porcine brain. Fresh porcine brain (First Link Ltd, Birmingham) was thawed in $100 \mathrm{ml}$ PBS per $50 \mathrm{~g}$ tissue at $37^{\circ} \mathrm{C}$. Once thawed, PBS was removed and substituted with buffer A (100 mM 2-( $N$-morpholino)ethanesulfonic acid, $1 \mathrm{mM}$ EGTA, $0.5 \mathrm{mM} \mathrm{MgCl}_{2}$ ) containing $2 \mathrm{mM}$ phenylmethylsulfonyl fluoride (PMSF). Homogenization was performed at $4{ }^{\circ} \mathrm{C}$ in a mixer, at pulse mode to avoid warming of the mixture. The homogenate was then centrifuged at $8000 \times g$ for $30 \mathrm{~min}$ at $4^{\circ} \mathrm{C}$ in a JA-17 rotor (Beckman Coulter) and supernatant filtered through two layers of gauze bandage to further remove particles. The final filtrate was pooled and centrifuged at $185,000 \times g$ for $60 \mathrm{~min}$ at $4{ }^{\circ} \mathrm{C}$ in a Ti-45 rotor (Beckman Coulter) to pellet CCVs. To further purify CCVs from impurities, the pellet was resuspended in a final volume of $12-15 \mathrm{ml}$ buffer A containing $2 \mathrm{mM} \mathrm{PMSF}$ and $0.02 \% \mathrm{NaN}_{3}$ and homogenized on ice for $5 \mathrm{~min}$ using a SS30 dounce stirrer (Stuart) at $125 \mathrm{rpm}$. An equivalent volume of a $12.5 \%$ Ficoll and $12.5 \%$ sucrose solution was added to the $\mathrm{CCV}$ homogenate and mixed gently. The mixture was then centrifuged at $30,000 \times g$ for $45 \mathrm{~min}$ at $4{ }^{\circ} \mathrm{C}$ using a JA-17 rotor (Beckman Coulter). CCV-containing supernatant was diluted fivefold with buffer A $+2 \mathrm{mM}$ PMSF and CCVs sedimented at $185,000 \times g$ for $60 \mathrm{~min}$ at $4{ }^{\circ} \mathrm{C}$ in a Ti-45 rotor (Beckman Coulter). The supernatant was discarded, vesicles resuspended in $4 \mathrm{ml}$ buffer $\mathrm{A}+2 \mathrm{mM}$ PMSF and $0.02 \% \mathrm{NaN}_{3}$, aliquoted and snapfrozen in liquid nitrogen, and stored at $-80^{\circ} \mathrm{C}$ until further use $e^{63}$.

Purification of triskelia from CCVs. Tris-extraction was performed according to ref. ${ }^{63}$ with slight modifications. Purified CCV were quickly thawed at $37^{\circ} \mathrm{C}$ and kept on ice. A solution of Tris pH 7.3, EDTA, protease inhibitor cocktail, and $\beta$ mercaptoethanol was added to the CCV suspension to obtain the final concentration of the Tris-extraction buffer $(0.5 \mathrm{M}$ Tris $\mathrm{pH}$ 7.3, $2 \mathrm{mM}$ EDTA, $1 \mathrm{mM} \beta$ mercaptoethanol and $1 \times$ protease inhibitors). The mix was left on ice for $10 \mathrm{~min}$ before ultracentrifugation in a TLA 100.3 rotor (Beckman Coulter) for $30 \mathrm{~min}$ at $220,000 \times g$ at $4^{\circ} \mathrm{C}$. Supernatant containing clathrin triskelia and adaptor proteins was further purified via size exclusion chromatography in buffer $\mathrm{B}(0.5 \mathrm{M}$ Tris $\mathrm{pH}$ 7.4, $2 \mathrm{mM}$ EDTA) using a Superose 6 increase 10/300 GL column (GE Healthcare) connected to an ÄKTA Purifier FPLC system (GE Healthcare) at a flow rate of $0.5 \mathrm{ml} / \mathrm{min}$ at $8{ }^{\circ} \mathrm{C}$. Elution fractions of $0.5 \mathrm{ml}$ volumes were collected and analyzed via SDS-PAGE. Clean clathrin fractions were pooled, and an equivalent volume of saturated $\mathrm{NH}_{4} \mathrm{SO}_{4}$ solution was carefully added. The mix was incubated on ice for $30 \mathrm{~min}$ to allow proteins precipitation and centrifuged for $30 \mathrm{~min}$ at $1600 \times \mathrm{g}$ using an Eppendorf $5810 \mathrm{R}$ centrifuge (Eppendorf) at $4^{\circ} \mathrm{C}$. The pellet was dissolved in 70-150 $\mu$ l extraction buffer (depending on pellet size), dialyzed in buffer B for $1 \mathrm{~h}$, and overnight at $4^{\circ} \mathrm{C}$ in buffer $\mathrm{C}(50 \mathrm{mM}$ Tris $\mathrm{pH} 8.0,50 \mathrm{mM} \mathrm{NaCl}, 2 \mathrm{mM}$ EDTA $1 \mathrm{mM} \beta$-mercaptoethanol, $1 \mathrm{mM}$ PMSF). Protein concentration $(3-5 \mathrm{mg} / \mathrm{ml})$ was determined by ultraviolet (UV) absorbance at $280 \mathrm{~nm}$, using an extinction coefficient of $1.0(\mathrm{mg} / \mathrm{ml}) / \mathrm{cm}$. Clathrin triskelia were either directly subjected to CLC dissociation (see below) or dialyzed against buffer A containing $2 \mathrm{mM} \mathrm{CaCl}_{2}$ overnight at $4{ }^{\circ} \mathrm{C}$ to allow cage assembly and long-term storage as native cages.

CLC dissociation and cage re-assembly. CLC dissociation was performed as previously described (Winkler and Stanley 1983). Clathrin triskelia solution were diluted in stripping buffer D (50 mM Tris pH $8.0,50 \mathrm{mM} \mathrm{NaCl}, 1.3 \mathrm{M} \mathrm{NaSCN}$, $2 \mathrm{mM}$ EDTA, $1 \mathrm{mM} \beta$-mercaptoethanol, $1 \mathrm{mM}$ PMSF) and subjected to size exclusion chromatography on a Superose 6 increase 10/300 GL column (GE Healthcare). Elution fractions were collected and analyzed via SDS-PAGE. Clean clathrin fractions were pooled, dialyzed twice against buffer $\mathrm{C}$ to remove NaSCN and concentrated by spin filtration using $10 \mathrm{~K}$ Amicon-Ultra centrifugal filters (Merck Millipore). Protein concentrations were determined by UV absorbance at $280 \mathrm{~nm}$ using an extinction coefficient of $1.0(\mathrm{mg} / \mathrm{ml}) / \mathrm{cm}$. To assemble CHC-only cages, CHC triskelia were dialyzed overnight in buffer A containing $2 \mathrm{mM} \mathrm{CaCl}_{2}$. To generate $\mathrm{CHC}-\mathrm{CLC}$ specific cages, $\mathrm{CHC}$-only cages were reconstituted with either CLCa or CLCb purified proteins in slight molar excess (CHC:CLC 1:1.2). Mix were incubated on ice for $1 \mathrm{~h}$, centrifuged for $30 \mathrm{~min}$ at $4{ }^{\circ} \mathrm{C}$ in a TLA55 rotor at $150,000 \times g$ and washed once with buffer A to eliminate the unbound CLCs.

Co-sedimentation of clathrin cages with myosin. Native and CLCa or CLCBonly cages were diluted to $1.5 \mu \mathrm{M}$ in buffer A $+0.1 \%$ Triton X-100 and $0.1 \mathrm{mg} / \mathrm{ml}$ BSA and mixed with $1.5 \mu \mathrm{M}$ of purified myosin $\mathrm{VI}^{998-1131}$ fragment on ice for $45 \mathrm{~min}$. Mix were then centrifuged at $4{ }^{\circ} \mathrm{C}$ for $30 \mathrm{~min}$ in a TLS-55 rotor at $108,000 \times g$, pellets were washed once with same volume of buffer $\mathrm{A}+0.1 \%$ Triton $\mathrm{X}-100$ and $0.1 \mathrm{mg} / \mathrm{ml} \mathrm{BSA}$ and centrifuged. Finally, pellets were dissolved in Laemmli buffer and analyzed through SDS-PAGE.

Negative staining of clathrin cages. Cages were adsorbed to freshly glow-discharged, carbon-coated formvar films on copper grids by placing grids on $10 \mu \mathrm{l}$ droplets of samples at $0.2 \mathrm{mg} / \mathrm{ml}$ on Parafilm for $90 \mathrm{~s}$. Excessive liquid was removed using filter paper. Grids were washed twice by sequentially transferring grids onto $15 \mu \mathrm{l}$ droplets of buffer A containing $2 \mathrm{mM} \mathrm{CaCl}_{2}$. Samples were then stained with $2 \%$ uranyl acetate in water for $1 \mathrm{~min}$ and air-dried before subjected to EM analysis. Specimens were observed using a Tecnai G2 (FEI) electron microscope at an acceleration voltage of $120 \mathrm{kV}$. Images were obtained using a SIS Morada digital camera and TIA software (FEI).

EM of Caco-2 cysts. Cysts grown in matrigel were fixed with $2.5 \%$ glutaraldehyde in $0.1 \mathrm{M}$ sodium cacodylate buffer $\mathrm{pH} 7.4$ for $1 \mathrm{~h}$ at RT, washed three times with cacodylate buffer, and fixed in $1 \%$ osmium tetroxide and $1.5 \%$ potassium ferrocyanide in $0.1 \mathrm{M}$ cacodylate for $1 \mathrm{~h}$ on ice. After several washes in distilled water, samples were en bloc stained with $0.5 \%$ uranyl acetate in water overnight at $4{ }^{\circ} \mathrm{C}$. Finally, samples were dehydrated in a graded ethanol series $(30 \%, 50 \%, 70 \%, 80 \%$, $90 \%, 96 \%, 20$ min each and 3 washes with absolute ethanol, 10 min each). Next, the samples were infiltrated in a 1:1 ethanol/Epon 812 solution for $2 \mathrm{~h}$, in 100\% Epoxy resin twice for $1 \mathrm{~h}$ each, and left overnight in resin before being embedded in resin and polymerized at $60^{\circ} \mathrm{C}$ for $48 \mathrm{~h}$. At the end of polymerization, the resin blocks were separated from the multiwell chamber and part of the block containing few cysts was mounted on a Leica Ultracut UCT ultramicrotome. Ultrathin $(70-90 \mathrm{~nm})$ sections were then collected on formvar carbon-coated slot grids and stained with uranyl acetate and Sato's lead citrate before imaged with a Talos $120 \mathrm{C}$ (FEI) electron microscope; images were acquired with a $4 \mathrm{k} \times 4 \mathrm{~K}$ Ceta CMOS camera.

For morphometrical analysis of the distribution of clathrin-coated structures, between 94 and 125 cellular profiles of well-polarized cells for each biological sample were imaged. Images of the CCPs present on the apical surface and connected to the membrane were acquired at the nominal magnification of $\times 36,000$ and classified as shallow, omega/constricted, or elongated according to their morphology. The distribution of clathrin-coated structures has been expressed as 
percentage among the different classes (Fig. 5e) or as the number per microns of apical membrane (Supplementary Fig. 10g). All statistical analyses were performed using GraphPad Prism. One-way analysis of variance was used to calculate statistical significance among different samples.

Protein expression and purification for NMR spectroscopy. GST-tagged myosin VI and CLCa were expressed separately in E. coli BL21 (DE3) cells (Thermo Fischer Scientific) at $17^{\circ} \mathrm{C}$ overnight upon reaching an $\mathrm{OD}_{600}$ value of $0.5-0.6$ by induction with $0.4 \mathrm{mM}$ IPTG. Cell pellets were resuspended in Buffer A $(50 \mathrm{mM}$ HEPES, $\mathrm{pH}$ 7.5, $300 \mathrm{mM} \mathrm{NaCl}, 1 \mathrm{mM}$ EDTA, 5\% glycerol, $2 \mathrm{mM}$ DTT, and Roche Complete Mini protease inhibitor cocktail), lysed by sonication, and the lysates were cleared via centrifugation. Supernatants were incubated with glutathione sepharose beads (GE Healthcare) for $3 \mathrm{~h}$ at $4{ }^{\circ} \mathrm{C}$ with a fresh protease inhibitor cocktail tablet added. Myosin VI-bound beads were washed twice with Buffer A, twice with Buffer B (PBS plus $500 \mathrm{mM} \mathrm{NaCl}, 0.1 \%$ Triton X-100, and $2 \mathrm{mM} \mathrm{DTT}$, pH 7.4), twice with Buffer C (PBS plus 2 mM DTT, pH 7.4), and once with Buffer D (50 mM Tris-Cl, $100 \mathrm{mM}$ $\mathrm{NaCl}, 1 \mathrm{mM}$ EDTA, $10 \%$ glycerol, and $2 \mathrm{mM}$ DTT, pH 7.0). The washed beads were resuspended in Buffer A and incubated with PreScission Protease (GE Healthcare) overnight at $4{ }^{\circ} \mathrm{C}$. Myosin VI was eluted with Buffer A, concentrated to $2 \mathrm{ml}$, purified via size exclusion chromatography on an FPLC system (Superdex75, GE Healthcare) with FPLC Buffer $\left(20 \mathrm{mM} \mathrm{NaPO}_{4}, 50 \mathrm{mM} \mathrm{NaCl}, 2 \mathrm{mM}\right.$ DTT, pH 6.5 ), and concentrated to $1.5 \mathrm{ml}$. CLCa-bound glutathione sepharose beads were washed five times with Buffer A, at which point purified myosin VI (from the above purification protocol) was added to the beads. The CLCa-myosin VI mixture was incubated with PreScission Protease overnight and eluted with Buffer A Elutions were concentrated to $2 \mathrm{ml}$, purified via size exclusion chromatography on an FPLC system with FPLC Buffer, and concentrated to $~ 300 \mu \mathrm{l}$.

NMR spectroscopy and backbone assignment. NMR data were collected at $10^{\circ} \mathrm{C}$ on Bruker 700 and $850 \mathrm{MHz}$ spectrometers. All experiments were conducted in 20 $\mathrm{mM} \mathrm{NaPO}_{4} \mathrm{pH} 6.5,50 \mathrm{mM} \mathrm{NaCl}, 2 \mathrm{mM}$ DTT, $0.1 \% \mathrm{NaN}_{3}, 1 \mathrm{mM}$ Pefabloc, and $5 \%$ $\mathrm{D}_{2} \mathrm{O}$. In all, $0.78 \mathrm{mM}{ }^{15} \mathrm{~N}$-labeled myosin VI and synthesized 5-FAM N-terminally tagged CLCa (GenScript) were used for the HSQC experiments acquired for CSP analysis. For structure determination, two separate samples were produced, namely, $0.4 \mathrm{mM}{ }^{13} \mathrm{C},{ }^{15} \mathrm{~N}$-labeled CLCa with equimolar unlabeled myosin VI, and $0.36 \mathrm{mM}{ }^{13} \mathrm{C},{ }^{15} \mathrm{~N}$-labeled myosin VI with equimolar unlabeled CLCa. For each sample, the following NMR experiments were performed: ${ }^{15} \mathrm{~N}-\mathrm{HSQC},{ }^{13} \mathrm{C}-\mathrm{HSQC}$, HNCO, HNCACB, ${ }^{15} \mathrm{~N}$-dispersed NOESY (120 ms mixing time), ${ }^{13} \mathrm{C}$-dispersed NOESY (100 ms mixing time), and ${ }^{13} \mathrm{C}$-dispersed-half-filtered-NOESY (100 ms mixing time). The NMR spectra were processed with NMRpipe ${ }^{64}$ and analyzed with XEASY ${ }^{65}$. Backbone assignments of myosin VI and CLCa in complex were done manually based on chemical shifts obtained previously on free myosin VI (BMRB 25544) and the HNCACB and HNCO spectra; the HNCACB spectrum showed both the intra-residue and proceeding residue $\mathrm{C} \alpha$ signals for most residues (Supplementary Fig. 4). Residues that were ambiguous were verified with HN-HN and $\alpha$-HN NOEs in the ${ }^{15}$ NOESY (Supplementary Fig. 5). Sidechain assignments were obtained by manual analysis of HNCACB, ${ }^{13} \mathrm{C}$-dispersed NOESY, and ${ }^{15} \mathrm{~N}$ dispersed NOESY experiments.

Structure determination and refinement. NOE assignments were done manually using ${ }^{13} \mathrm{C}$-dispersed NOESY, ${ }^{15} \mathrm{~N}$-dispersed NOESY, and ${ }^{13} \mathrm{C}$-dispersed half-filtered NOESY experiments. Chemical shifts for backbone carbons and amide nitrogen, amide hydrogen, $\mathrm{H} \alpha$, and $\mathrm{C} \beta$ from myosin VI and CLCa were used with TALOS ${ }^{66}$ to obtain backbone $\phi$ and $\psi$ torsion angle restraints. Structure calculations were performed with Xplor-NIH (version 2.47$)^{67}$ by using standard scripts. Twenty linear starting structures were subjected to 35,000 simulated annealing steps followed by 5000 cooling steps, both of 0.005 ps. The lowest energy structure from this set was used as the starting structure for refinement closely following the protocol described in ref. ${ }^{68}$ but with a temperature step of $1 \mathrm{~K}$ used during simulated annealing. Constraints used during the structure calculations include intramolecular and intermolecular NOE restraints, dihedral angle restraints, and backbone hydrogen bonds in helical regions. A summary of the data used for the structure calculations is provided in Table 1. Hydrogen bond distances between the acceptor oxygen and donor hydrogen and nitrogen atoms were set to 1.81-2.09 and 2.71-3.13 $\AA$, respectively. Structures and NOE assignments were checked manually to identify errors, corrections were made if necessary, and structures recalculated; this process was done iteratively. Of the 300 final refined structures calculated, the 20 lowest energy structures without distance violations $>0.5 \AA$ and dihedral angle violations $>5^{\circ}$ were selected to represent the structure of myosin VI in complex with CLCa. Pymol (Schrödinger) was used for visualization and figure generation; MOLMOL ${ }^{69}$ was used for r.m.s.d. calculation and figure generation. Among the 20 lowest energy structures, no amino acid was disfavored by Ramachandran analyses; 1,6 , and $94 \%$ were in the generously allowed, allowed, and favored regions, respectively.

Reporting summary. Further information on research design is available in the Nature Research Reporting Summary linked to this article.

\section{Data availability}

Data supporting the findings of this manuscript are available in the Source Data file. All uncropped immunoblots associated with Figs. 1b, 1c, 1d, 1e, 2b,4f, 4d, 5a, 6b, 6c and Supplementary Figs. 1a, 1c, 2d, 9a, 9b, 10a,10c, 11c, 11d, 11e, 12a, 12b are provided as Source Data file. A reporting summary for this Article is available as a Supplementary Information file. Chemical shift assignments for the complex of myosin VI ${ }^{1050-1131}$ and CLCa ${ }^{46-61}$ have been deposited in the Biological Magnetic Resonance Bank under the ID code 30500, and the atomic coordinates for the complex have been deposited in the Protein Data Bank under the ID code 6E5N.

Received: 3 June 2019; Accepted: 27 September 2019; Published online: 31 October 2019

\section{References}

1. Brodsky, F. M. Diversity of clathrin function: new tricks for an old protein. Annu. Rev. Cell Dev. Biol. 28, 309-336 (2012).

2. Kirchhausen, T., Owen, D. \& Harrison, S. C. Molecular structure, function, and dynamics of clathrin-mediated membrane traffic. Cold Spring Harb. Perspect. Biol. 6, a016725 (2014)

3. Ferreira, F. et al. Endocytosis of G protein-coupled receptors is regulated by clathrin light chain phosphorylation. Curr. Biol. 22, 1361-1370 (2012).

4. Poupon, V. et al. Clathrin light chains function in mannose phosphate receptor trafficking via regulation of actin assembly. Proc. Natl Acad. Sci. USA 105, 168-173 (2008).

5. Wu, S. et al. Clathrin light chains' role in selective endocytosis influences antibody isotype switching. Proc. Natl Acad. Sci. USA 113, 9816-9821 (2016)

6. Boulant, S., Kural, C., Zeeh, J. C., Ubelmann, F. \& Kirchhausen, T. Actin dynamics counteract membrane tension during clathrin-mediated endocytosis. Nat. Cell Biol. 13, 1124-1131 (2011).

7. Chen, C. Y. \& Brodsky, F. M. Huntingtin-interacting protein 1 (Hip1) and Hip1-related protein (Hip1R) bind the conserved sequence of clathrin light chains and thereby influence clathrin assembly in vitro and actin distribution in vivo. J. Biol. Chem. 280, 6109-6117 (2005).

8. Wilbur, J. D. et al. Conformation switching of clathrin light chain regulates clathrin lattice assembly. Dev. Cell 18, 841-848 (2010).

9. Majeed, S. R. et al. Clathrin light chains are required for the gyrating-clathrin recycling pathway and thereby promote cell migration. Nat. Commun. 5, 3891 (2014).

10. Bonazzi, M. et al. Clathrin phosphorylation is required for actin recruitment at sites of bacterial adhesion and internalization. J. Cell Biol. 195, 525-536 (2011).

11. Cossart, P. \& Helenius, A. Endocytosis of viruses and bacteria. Cold Spring Harb. Perspect. Biol. 6, a016972 (2014).

12. Cureton, D. K., Massol, R. H., Whelan, S. P. \& Kirchhausen, T. The length of vesicular stomatitis virus particles dictates a need for actin assembly during clathrin-dependent endocytosis. PLoS Pathog. 6, e1001127 (2010).

13. Buss, F., Spudich, G. \& Kendrick-Jones, J. Myosin VI: cellular functions and motor properties. Annu. Rev. Cell Dev. Biol. 20, 649-676 (2004).

14. Bonazzi, M. et al. A common clathrin-mediated machinery co-ordinates cellcell adhesion and bacterial internalization. Traffic 13, 1653-1666 (2012).

15. Tsygankova, O. M. \& Keen, J. H. A unique role for clathrin light chain A in cell spreading and migration. J. Cell Sci. 132, jcs224030 (2019).

16. Maib, H., Ferreira, F., Vassilopoulos, S. \& Smythe, E. Cargo regulates clathrincoated pit invagination via clathrin light chain phosphorylation. J. Cell Biol. 217, 4253-4266 (2018).

17. Chen, P. H. et al. Crosstalk between CLCb/Dyn1-mediated adaptive clathrinmediated endocytosis and epidermal growth factor receptor signaling increases metastasis. Dev. Cell 40, 278.e5-288.e5 (2017).

18. Wollscheid, H. P. et al. Diverse functions of myosin VI elucidated by an isoform-specific alpha-helix domain. Nat. Struct. Mol. Biol. 23, 300-308 (2016).

19. Hegan, P. S., Giral, H., Levi, M. \& Mooseker, M. S. Myosin VI is required for maintenance of brush border structure, composition, and membrane trafficking functions in the intestinal epithelial cell. Cytoskeleton (Hoboken) 69, 235-251 (2012).

20. Liu, S. H., Wong, M. L., Craik, C. S. \& Brodsky, F. M. Regulation of clathrin assembly and trimerization defined using recombinant triskelion hubs. Cell 83, 257-267 (1995).

21. Ybe, J. A. et al. Clathrin self-assembly is regulated by three light-chain residues controlling the formation of critical salt bridges. EMBO J. 17, 1297-1303 (1998).

22. Brodsky, F. et al. Clathrin light chains: arrays of protein motifs that regulate coated-vesicle dynamics. Trends Biochem. Sci. 16, 208-213 (1991).

23. Fotin, A. et al. Molecular model for a complete clathrin lattice from electron cryomicroscopy. Nature 432, 573-579 (2004). 
24. Walters, K. J. et al. Characterizing protein-protein complexes and oligomers by nuclear magnetic resonance spectroscopy. Methods Enzymol. 339, 238-258 (2001).

25. Sahlender, D. A. et al. Optineurin links myosin VI to the Golgi complex and is involved in Golgi organization and exocytosis. J. Cell Biol. 169, 285-295 (2005).

26. Spudich, G. et al. Myosin VI targeting to clathrin-coated structures and dimerization is mediated by binding to Disabled-2 and PtdIns(4,5)P2. Nat. Cell Biol. 9, 176-183 (2007).

27. He, F. et al. Myosin VI contains a compact structural motif that binds to ubiquitin chains. Cell Rep. 14, 2683-2694 (2016).

28. Buss, F., Luzio, J. P. \& Kendrick-Jones, J. Myosin VI, a new force in clathrin mediated endocytosis. FEBS Lett. 508, 295-299 (2001).

29. Buss, F. et al. Myosin VI isoform localized to clathrin-coated vesicles with a role in clathrin-mediated endocytosis. EMBO J. 14, 3676-3684 (2001).

30. Biemesderfer, D., Mentone, S. A., Mooseker, M. \& Hasson, T. Expression of myosin VI within the early endocytic pathway in adult and developing proximal tubules. Am. J. Physiol. Ren. Physiol. 282, F785-F794 (2002).

31. Jaffe, A. B., Kaji, N., Durgan, J. \& Hall, A. Cdc42 controls spindle orientation to position the apical surface during epithelial morphogenesis. J. Cell Biol. 183, 625-633 (2008).

32. Damke, H., Baba, T., Warnock, D. E. \& Schmid, S. L. Induction of mutant dynamin specifically blocks endocytic coated vesicle formation. J. Cell Biol. 127, 915-934 (1994).

33. Engqvist-Goldstein, A. E. et al. The actin-binding protein Hip1R associates with clathrin during early stages of endocytosis and promotes clathrin assembly in vitro. J. Cell Biol. 154, 1209-1223 (2001).

34. Legendre-Guillemin, V. et al. HIP1 and HIP12 display differential binding to F-actin, AP2, and clathrin. Identification of a novel interaction with clathrin light chain. J. Biol. Chem. 277, 19897-19904 (2002).

35. Henry, K. R. et al. Scd5p and clathrin function are important for cortical actin organization, endocytosis, and localization of sla2p in yeast. Mol. Biol. Cell 13, 2607-2625 (2002).

36. Aschenbrenner, L., Lee, T. \& Hasson, T. Myo6 facilitates the translocation of endocytic vesicles from cell peripheries. Mol. Biol. Cell 14, 2728-2743 (2003).

37. Wagner, W. et al. Myosin VI drives clathrin-mediated AMPA receptor endocytosis to facilitate cerebellar long-term depression. Cell Rep. 28, 11. e9-20.e9 (2019).

38. Wells, A. L. et al. Myosin VI is an actin-based motor that moves backwards. Nature 401, 505-508 (1999).

39. DeLuca-Flaherty, C., McKay, D. B., Parham, P. \& Hill, B. L. Uncoating protein (hsc70) binds a conformationally labile domain of clathrin light chain LCa to stimulate ATP hydrolysis. Cell 62, 875-887 (1990).

40. Batters, C., Brack, D., Ellrich, H., Averbeck, B. \& Veigel, C. Calcium can mobilize and activate myosin-VI. Proc. Natl Acad. Sci. USA 113, E1162-E1169 (2016).

41. Engqvist-Goldstein, A. E. et al. RNAi-mediated Hip1R silencing results in stable association between the endocytic machinery and the actin assembly machinery. Mol. Biol. Cell 15, 1666-1679 (2004).

42. Huang, F., Khvorova, A., Marshall, W. \& Sorkin, A. Analysis of clathrinmediated endocytosis of epidermal growth factor receptor by RNA interference. J. Biol. Chem. 279, 16657-16661 (2004).

43. Ripoll, L. et al. Myosin VI and branched actin filaments mediate membrane constriction and fission of melanosomal tubule carriers. J. Cell Biol. 217, 2709-2726 (2018).

44. Mund, M. et al. Systematic nanoscale analysis of endocytosis links efficient vesicle formation to patterned actin nucleation. Cell 174, 884-896 e17 (2018).

45. Collins, A., Warrington, A., Taylor, K. A. \& Svitkina, T. Structural organization of the actin cytoskeleton at sites of clathrin-mediated endocytosis. Curr. Biol. 21, 1167-1175 (2011).

46. Swiatecka-Urban, A. et al. Myosin VI regulates endocytosis of the cystic fibrosis transmembrane conductance regulator. J. Biol. Chem. 279, 38025-38031 (2004).

47. Blaine, J. et al. PTH-induced internalization of apical membrane NaPi2a: role of actin and myosin VI. Am. J. Physiol. Cell Physiol. 297, C1339-C1346 (2009).

48. Ameen, N. \& Apodaca, G. Defective CFTR apical endocytosis and enterocyte brush border in myosin VI-deficient mice. Traffic 8, 998-1006 (2007).

49. Gotoh, N. et al. Altered renal proximal tubular endocytosis and histology in mice lacking myosin-VI. Cytoskeleton (Hoboken) 67, 178-192 (2010).

50. Collaco, A., Jakab, R., Hegan, P., Mooseker, M. \& Ameen, N. Alpha-AP-2 directs myosin VI-dependent endocytosis of cystic fibrosis transmembrane conductance regulator chloride channels in the intestine. J. Biol. Chem. 285, 17177-17187 (2010).

51. Chen, T. et al. Myosin VI mediates the movement of NHE3 down the microvillus in intestinal epithelial cells. J. Cell Sci. 127, 3535-3545 (2014).

52. Skruzny, M. et al. Molecular basis for coupling the plasma membrane to the actin cytoskeleton during clathrin-mediated endocytosis. Proc. Natl Acad. Sci. USA 109, E2533-E2542 (2012).
53. Clarke, N. I. \& Royle, S. J. FerriTag is a new genetically-encoded inducible tag for correlative light-electron microscopy. Nat. Commun. 9, 2604 (2018).

54. Messa, M. et al. Epsin deficiency impairs endocytosis by stalling the actindependent invagination of endocytic clathrin-coated pits. Elife 3, e03311 (2014).

55. Garcia-Alai, M. M. et al. Epsin and Sla2 form assemblies through phospholipid interfaces. Nat. Commun. 9, 328 (2018)

56. Sochacki, K. A., Dickey, A. M., Strub, M. P. \& Taraska, J. W. Endocytic proteins are partitioned at the edge of the clathrin lattice in mammalian cells. Nat. Cell Biol. 19, 352-361 (2017).

57. Antonny, B. et al. Membrane fission by dynamin: what we know and what we need to know. EMBO J. 35, 2270-2284 (2016)

58. Mettlen, M., Chen, P. H., Srinivasan, S., Danuser, G. \& Schmid, S. L. Regulation of clathrin-mediated endocytosis. Annu Rev. Biochem 87, 871-896 (2018).

59. Yu, C. et al. Myosin VI undergoes cargo-mediated dimerization. Cell 138, 537-548 (2009).

60. Taylor, M. J., Perrais, D. \& Merrifield, C. J. A high precision survey of the molecular dynamics of mammalian clathrin-mediated endocytosis. PLoS Biol. 9, e1000604 (2011).

61. Jackson, A. P. \& Parham, P. Structure of human clathrin light chains. Conservation of light chain polymorphism in three mammalian species. $J$. Biol. Chem. 263, 16688-16695 (1988).

62. Eletr, Z. M., Huang, D. T., Duda, D. M., Schulman, B. A. \& Kuhlman, B. E2 conjugating enzymes must disengage from their E1 enzymes before E3dependent ubiquitin and ubiquitin-like transfer. Nat. Struct. Mol. Biol. 12, 933-934 (2005).

63. Ungewickell, E. \& Ungewickell, H. Bovine brain clathrin light chains impede heavy chain assembly in vitro. J. Biol. Chem. 266, 12710-12714 (1991).

64. Schaefer, A. W. et al. L1 endocytosis is controlled by a phosphorylationdephosphorylation cycle stimulated by outside-in signaling by L1. J. Cell Biol. 157, 1223-1232 (2002).

65. Bartels, C., Xia, T. H., Billeter, M., Guntert, P. \& Wuthrich, K. The program XEASY for computer-supported NMR spectral analysis of biological macromolecules. J. Biomol. NMR 6, 1-10 (1995)

66. Cornilescu, G., Delaglio, F. \& Bax, A. Protein backbone angle restraints from searching a database for chemical shift and sequence homology. J. Biomol. NMR 13, 289-302 (1999).

67. Schwieters, C. D., Kuszewski, J. J., Tjandra, N. \& Clore, G. M. The Xplor-NIH NMR molecular structure determination package. J. Magn. Reson. 160, 65-73 (2003).

68. Schwieters, C. D., Bermejo, G. A. \& Clore, G. M. Xplor-NIH for molecular structure determination from NMR and other data sources. Protein Sci. 27, 26-40 (2018)

69. Koradi, R., Billeter, M. \& Wuthrich, K. MOLMOL: a program for display and analysis of macromolecular structures. J Mol. Graph 14, 51-55, 29-32 (1996).

\section{Acknowledgements}

We thank Tom Kirchhausen, Stephen Royle, Klemens Rottner, and Elizabeth Smythe for DNA constructs. We thank ALEMBIC facility at San Raffaele Scientific Institute, Milan, Italy for support in electron microscopic analysis and the Biochemistry and Structural Biology Unit at the European Institute of Oncology for support with isothermal titration calorimetric analysis. This work was supported by the Associazione Italiana per la Ricerca sul Cancro (AIRC IG19875 to S.P.); the Italian Ministry of Education, Universities and Research (PRIN 20108MXN2J to S.P.); the Intramural Research Program through the CCR, NCI, NIH (1ZIABC011627 to K.J.W.); and Wellcome Trust Investigator Award (107858/Z/15/Z to F.M.B.). C.A.N.'s work is supported by the Marie Skłodowska-Curie Actions (MSCA-IF-2016 \#752553). M.B. was and R.S.d.P. is a PhD student within the European School of Molecular Medicine (SEMM). M.B was supported by a fellowship from Fondazione Umberto Veronesi (FUV) and by an EMBO short-term travel fellowship.

\section{Author contributions}

M.B. performed most of the biochemical experiments and contributed to result interpretation; G.R.B. performed the NMR experiments and solved the structures; C.A.N generated the genetically reconstituted Caco- 2 cells and cysts and contributed to experimental design; E.M. performed the Hip1R experiments; R.S.d.P. prepared the samples for EM analysis; A.R. conducted the EM experiments; L.R. supervised the mice experiments; J.W. performed the ITC experiments. F.M.B. generated the CLC KO mice and contributed to experimental design and paper writing; K.J.W. supervised the NMR analysis and contributed to NMR interpretation and paper writing; S.P. coordinated the team, designed the experiments, and wrote the paper.

\section{Competing interests}

The authors declare no competing interests. 


\section{Additional information}

Supplementary information is available for this paper at https://doi.org/10.1038/s41467019-12855-6.

Correspondence and requests for materials should be addressed to F.M.B., K.J.W. or S.P.

Peer review information Nature Communications thanks the anonymous reviewers for their contribution to the peer review of this work. Peer reviewer reports are available.

Reprints and permission information is available at http://www.nature.com/reprints

Publisher's note Springer Nature remains neutral with regard to jurisdictional claims in published maps and institutional affiliations. (c) Open Access This article is licensed under a Creative Commons Attribution 4.0 International License, which permits use, sharing, adaptation, distribution and reproduction in any medium or format, as long as you give appropriate credit to the original author(s) and the source, provide a link to the Creative Commons license, and indicate if changes were made. The images or other third party material in this article are included in the article's Creative Commons license, unless indicated otherwise in a credit line to the material. If material is not included in the article's Creative Commons license and your intended use is not permitted by statutory regulation or exceeds the permitted use, you will need to obtain permission directly from the copyright holder. To view a copy of this license, visit http://creativecommons.org/licenses/by/4.0/.

(C) The Author(s) 2019 